\title{
A Compressor for Effective Archiving, Retrieval, and Updating of XML Documents
}

\author{
JUN-KI MIN, MYUNG-JAE PARK, and CHIN-WAN CHUNG \\ Korea Advanced Institute of Science and Technology
}

\begin{abstract}
Like HTML, many XML documents are resident on native file systems. Since XML data is irregular and verbose, the disk space and the network bandwidth are wasted. To overcome the verbosity problem, the research on compressors for XML data has been conducted. Some XML compressors do not support querying compressed data, while other XML compressors which support querying compressed data blindly encode tags and data values using predefined encoding methods. Existing XML compressors do not provide the facility for updates on compressed XML data.

In this paper, we propose XPRESS, an XML compressor which supports direct updates and efficient evaluations of queries on compressed XML data. XPRESS adopts a novel encoding method, called reverse arithmetic encoding, which is intended for encoding label paths of XML data, and applies diverse encoding methods depending on the types of data values. Experimental results with real-life data sets show that XPRESS achieves significant improvements on query performance for compressed XML data and reasonable compression ratios. On the average, the query performance of XPRESS is 2.13 times better than that of an existing XML compressor and the compression ratio of XPRESS is about $71 \%$. Additionally, we demonstrate the efficiency of the updates performed directly on compressed XML data.
\end{abstract}

Categories and Subject Descriptors: I.7.1 [Document and Text Processing]: Document and Text Editing - Document management; I.7.2 [Document and Text Processing]: Document Preparation-Markup languages; XML

General Terms: Algorithms, Management, Performance

Additional Key Words and Phrases: Compression, Query Processing, XML

\section{INTRODUCTION}

The eXtensible Markup Language (XML) [Bray et al. 1998] is intended as a markup language for an arbitrary document structure, as opposed to HTML which is a markup language for a specific kind of hypertext data.

The basic data model of XML is a labeled tree, where each element or attribute is represented as a node in the tree, and its tag corresponds to the label of the corresponding node. This tree structured data model is simple enough to devise

A preliminary version of this paper appeared in the Proceedings of the 2003 ACM SIGMOD International Conference on Management of Data, Jun. 9-12, 2003, pp. 122-133.

Author's address: JUN-KI MIN, MYUNG-JAE PARK, and CHIN-WAN CHUNG, Division of Computer Science, Department of Electrical Engineering \& Computer Science, Korea Advanced Institute of Science and Technology (KAIST), 373-1 Guseong-dong, Yuseong-gu, Daejeon, 305-701, KOREA; email: jkmin@islab.kaist.ac.kr; jpark@islab.kaist.ac.kr; chungcw@islab.kaist.ac.kr.

Permission to make digital/hard copy of all or part of this material without fee for personal or classroom use provided that the copies are not made or distributed for profit or commercial advantage, the ACM copyright/server notice, the title of the publication, and its date appear, and notice is given that copying is by permission of the ACM, Inc. To copy otherwise, to republish, to post on servers, or to redistribute to lists requires prior specific permission and/or a fee.

(C) $20 \mathrm{YY}$ ACM 1533-5399/20YY/0800-0001 $\$ 5.00$ 
efficient as well as elegant algorithms for it. Due to its flexibility and simplicity, XML is rapidly emerging as the de facto standard for exchanging and querying documents on the web required for the next generation web applications including electronic commerce and intelligent web searching.

To retrieve XML data, XML query languages such as XPath [Clark and DeRose 1999] and XQuery [Boag et al. 2002] have been proposed recently. These languages are based on path expressions to traverse irregularly structured data. Therefore, the efficient support of path expressions over XML data is a major issue in the field of XML [Goldman and Widom 1997; Grust 2002].

Currently, a variety of research for XML data has focused on issues related to XML storage [Florescu and Kossman 1999], retrieval [Goldman and Widom 1997; Fernandez and Suciu 1998], and publication [Fernandez et al. 2000; Shanmugasundaram et al. 2000]. Although some XML data are managed in the XML storage, large portions of XML data are still on native file systems as in the case of HTML. Thus, in order for XML to become the true internet standard, the research on the efficient management of the file based XML data is required.

One of the interesting applications for file based XML data is web searching. In this application, if each web server manages its own data in the form of XML and transmits it through the network, the storage and the network bandwidth are wasted since XML data is irregular and verbose. To overcome the verbosity problem, the research on compressors for XML data has been conducted [Liefke and Suciu 2000; Tolani and Haritsa 2002; Cheng and Ng 2004; Arion et al. 2004].

XMill [Liefke and Suciu 2000] was designed to minimize the size of compressed XML data. However, XMill was not intended to support querying compressed XML data.

XGrind [Tolani and Haritsa 2002] was devised to evaluate queries directly on compressed XML data. However, the encoding scheme of XGrind does not sufficiently take into account the properties of XML data and query languages.

Furthermore, XMill and XGrind do not support direct updates on compressed XML data. Thus, to update the XML data, the compressed XML data should be decompressed completely. And then, updates and recompression are performed. This approach consumes much time.

\subsection{Our Contributions}

In this paper, we propose XPRESS, an XML compressor, to compress XML data for the purposes of archiving, retrieving and exchanging. XPRESS supports direct updates and efficient evaluations of queries on compressed XML data.

In contrast to the web search engines for HTML, XML search engines can use structural predicates such as path expressions for search conditions since XML differentiates the structure from contents. For example, if users want to select XML files that contain some information about sales of houses, users can submit a search condition like " $\exists(/ /$ sales/house $)$ ".

To perform the kinds of queries as mentioned above on the compressed data in XMill, a complete decompression is required. In XGrind, although the overhead for the complete decompression is removed, the overhead of maintenance and evaluation of the simple path to each element, similar to that for uncompressed XML data, still remains. In contrast to the other XML compressors, XPRESS gets rid of 
this overhead by using a novel encoding method, called reverse arithmetic encoding, and minimizes the overhead of partial decompression by utilizing diverse encoding methods.

Existing XML compressors do not provide the facility for update on compressed XML data. To our best knowledge, XPRESS is the first XML compressor which supports direct update on compressed XML data. The current implementation of XPRESS supports only point update in which the updating point is specified by a path expression.

XPRESS has the following novel combination of characteristics to compress, retrieve, and update XML data efficiently.

- Reverse Arithmetic Encoding: Since existing XML compressors simply represent each tag by using a unique identifier, they are inefficient to handle path expressions on compressed XML data. In contrast, XPRESS adopts the reverse arithmetic encoding method that encodes a label path as a distinct interval in $[0.0,1.0)$. Using the containment relationships among the intervals, path expressions are evaluated on compressed XML data efficiently.

-Automatic Type Inference: Some XML compressors compact data values of XML elements by using predefined encoding methods (e.g., huffuman encoding). However, according to the types of data values, the kinds of efficient encoding methods are different. In some XML compressors, the types of data values are manually interpreted. Thus, if there is no human interference, data values of XML elements and attributes are not compressed properly. In XPRESS, to apply effective encoding methods to various kinds of data values of XML elements, we devise an efficient type inference engine that does not require the human interference.

- Apply Diverse Encoding Methods to Different Types: According to the inferred type information, we apply proper encoding methods to data values. Thus, we achieve a high compression ratio and minimize the overhead of partial decompression in the query processing phase.

- Support of Direct Update: To update compressed XML data, existing XML compressors should perform the complete decompression. But, by analyzing the portion of the XML data to be inserted, XPRESS performs the partial decompression of the compressed XML data. Thus, XPRESS supports direct updates on the compressed XML data without the complete decompression and recompression.

- Semi-adaptive Approach: Our compression scheme is categorized as the semiadaptive approach [Howard and Vitter 1991] which uses a preliminary scan of the input file to gather statistics. Since the semi-adaptive approach does not change the statistics during the compression phase, the encoding rules for data are independent to the locations of data. This property allows us to query compressed XML data directly.

- Homomorphic Compression: Like XGrind, XPRESS is a homomorphic compressor which preserves the structure of the original XML data in compressed XML data. Thus, XML segmentations that satisfy given query conditions are efficiently extracted. 
We implemented XPRESS and conducted an extensive experimental study with real-life XML data sets. In our experiment, XPRESS demonstrates significantly improved query performance and reasonable compression ratio compared to the other XML compressors. In addition, to show the efficiency of the updates, we compared the update performance of XPRESS with a naive approach. On the average, the query performance of XPRESS is 2.13 times better than that of an existing XML compressor and the compression ratio of XPRESS is $71 \%$.

\subsection{Organization}

The remainder of the paper is organized as follows. In Section 2, we present general purpose compression methods and compression tools for XML data. In Section 3, we present the features of XPRESS. Section 4 describes the compression techniques of XPRESS. Section 5 provides the update processing technique of XPRESS in detail. Section 6 contains the result of our experiments which compares the performance of XPRESS to those of the other XML compressors. Finally, in Section 7, we summarize our work and suggest some future studies.

\section{RELATED WORK}

The data compression has a long and rich history in the field of information theory [Shannon 1948; Huffman 1952].

One advantage of data compression is that the required disk space of data can be reduced significantly. The second advantage is the saving of the network bandwidth. Since the overall size of data is decreased, much more data can be transferred through the network within a given period of time. Another advantage is that data compression improves the overall performance of database systems. By compressing data, more information can be loaded in the buffer and the number of disk I/Os is reduced. Therefore, the performance of the system is enhanced.

\subsection{General Purpose Compression}

According to the ability of data recovery, compression methods are classified into two groups: the lossy compression and the lossless compression.

The lossy compression reduces a file by permanently eliminating certain information. The data compressed by the lossy compression cannot be reconstructed into the original data by the decompression. Thus, in this paper, we do not address the lossy compression since the lossless recovery is required for textual information.

The lossless compression is categorized into three groups: static, semi-adaptive, adaptive [Howard and Vitter 1991]. The static compression uses fixed statistics or does not use any statistics. The semi-adaptive compression scans the input data to gather statistics preliminarily and rescans the data to compress. The adaptive compression does not require any prior statistics. Instead, statistics are gathered dynamically, and updated during the compression phase.

The representative compression methods of the static compression are dictionary encoding, binary encoding and differential encoding.

The dictionary encoding method assigns an integer value to each new word from the input data so that each word in the input data can be compressed by using a uniquely assigned integer value. Some special types of data such as numeric data can be encoded in binary, e.g., integer or floating. This is called the binary 
encoding method. The differential encoding method, also called delta encoding, replaces a data item with a code value that defines its relationship to a specific data item. For example, a data sequence of 1500, 1520, 1600, 1550 will be encoded as $1500,20,100,50$.

Since the static approach does not consider the nature of given data, compression ratios are quite different depending on the input data. Thus, it is important to adopt proper encoding methods on account of data properties.

In the semi-adaptive compression methods, huffman encoding [Huffman 1952] and arithmetic encoding [Witten et al. 1987] are the examples.

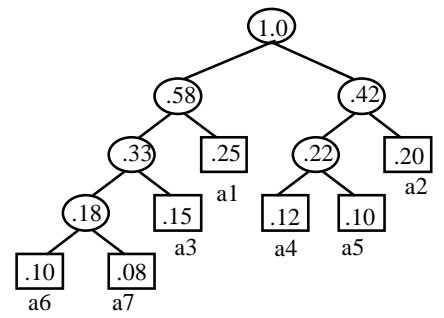

Fig. 1. An example of the huffman tree

The basic idea of the huffman encoding method is to assign shorter codes to more frequently appearing symbols and longer codes to less frequently appearing symbols. To assign a code to each character, a binary tree, called the huffman tree, is constructed using the statistics gathered by a preliminary scan. A simple example of the huffman tree is shown in Figure 1. The leaf nodes of the huffman tree are assigned symbols in input data. The value in a leaf node is the frequency of the symbol. The left edges of the huffman tree are labeled with 0 and the right edges are labeled with 1 so that the code assigned to each symbol is the sequence of labels starting from the root to the leaf node of the symbol. The codes generated by huffman encoding do not keep the order information among symbols.

The arithmetic encoding method represents a given message by choosing any number from a calculated interval. Symbols are assigned disjoint intervals according to their frequencies. Using the order of intervals for symbols, the order information among symbols is preserved. Successive symbols of a message reduce the length of the interval of the first symbol in accordance with the frequencies of the symbols. After reducing the length of the interval by applying all the symbols of the message, the message is transformed into a variable length bit string that represents any number within the reduced interval.

In the adaptive compression, adaptive huffman encoding, adaptive arithmetic encoding, and $L Z$ encoding are representatives.

The adaptive huffman encoding method and the adaptive arithmetic encoding method are similar to the huffman encoding method and the arithmetic encoding method, respectively. However, the adaptive huffman encoding method dynamically construct the huffman tree, and the adaptive arithmetic encoding method calculates the intervals by gathering frequencies and probabilities dynamically. In other words, these adaptive encoding methods dynamically update statistics (i.e., huffman tree or 
intervals) of each symbol based on the previous statistics during compression phase, instead of using the predefined statistics used in the semi-adaptive compression methods.

The LZ (Lempel-Ziv) encoding method, similar to the dictionary encoding method of the static compression, records the string seen previously. When the new string is read, the LZ encoder finds the longest common substring in the string seen previously, then converts the new string into a pointer to the common substring with an additional string. (see more details in [Salomon 1998]).

\subsection{XML Compression}

Recently, some research on compressors for XML data have been conducted [Arion et al. 2004; Cheng and Ng 2004; Liefke and Suciu 2000; Tolani and Haritsa 2002].

XMill [Liefke and Suciu 2000] physically separates XML tags and attributes from their data values and groups semantically related data values into containers. XML tags and attributes are compressed by the dictionary encoding method.

Each container can be compressed by a user specified encoding method if the user wants to apply specified encoding method. In order to apply specialized compressors to containers, a human interpretation of the containers is required. Finally, each compressed container is recompressed by a build-in library, called zlib. XMill is intended to minimize the size of compressed XML data. However, XMill does not support direct querying on compressed XML data.

A distinguishable feature of XGrind [Tolani and Haritsa 2002] compared to XMill is that it supports querying compressed XML data. In XGrind, data values are compressed by huffman encoding or dictionary encoding and tags are compressed by dictionary encoding. Using DTD, XGrind determines to apply huffman encoding or dictionary encoding for a certain attribute value. In XGrind, to evaluate a path expression, whenever an element is visited by the query processor, the identifier sequence which represents the label path from the root element to the currently visited element is found and the query processor checks whether this identifier sequence satisfies the path expression. In addition, to evaluate range queries on compressed XML data, a partial decompression is always required since huffman encoding and dictionary encoding do not preserve any order information among data items.

Recently, XQueC [Arion et al. 2004] and XQzip [Cheng and Ng 2004] are proposed for the queriable XML compression. Like XMill, these compressors separate the structure and data values. To speed up the query performance, these compressors utilize the path indexes such as dataguide, and structural index tree (SIT).

The work of XQueC is originated from the compressed database systems [Chen et al. 2000]. Unlike other XML compressors, XQueC uses a database as the storage system. Thus, in XQueC, the compressed fragments of XML data are scattered over databases and the compressed XML file is not generated. Therefore, the reconstruction is needed for XQueC to transmit the compressed XML data through the network.

In XQzip, the structural information of XML data is maintained by the SIT which is a variation of path indexes and data values are compressed by the built-in library, zlib. Although, the data values are separated into a sequence of blocks, the (partial/full) decompression of compressed data values are required in XQzip. In 
addition, XQzip does not support the order based predicates since SIT does not reflect the document order exactly.

Furthermore, none of them supports direct updates on compressed XML data. Thus, to update XML data, a complete decompression and recompression are required.

Besides XML compression, to speed up the query performance, many effective methods such as binary XML encoding [Bayardo et al. 2004] and the KoN pattern tree [Zhang et al. 2004] have been proposed. These methods are focused on effective physical structures for XML data.

\section{FEATURES OF XPRESS}

In this section, we present the major features of XPRESS which support effective query processing on compressed XML data.

In our paper, we do not treat attributes and elements differently since attributes in XML data are considered as specific elements. The prefix '@' is added to an attribute name to distinguish attributes and elements.

Among various XML query languages, XPath [Clark and DeRose 1999] is simple, but powerful enough to address any part of an XML document. Thus, we present a subset of XPath which XPRESS supports.

An XPath expression consists of a sequence of steps. A step is applied to a single node (the context node) and generates the result nodes. At the beginning of an XPath expression, the context node is not an element node, but the root node of an XML tree. A result node from each step is used as the context node for the following step.

For the convenience of usage, W3C also provides the abbreviated syntax of XPath which is more popular than the full syntax of XPath.

Figure 2 provides the BNF for the subset of XPath which we consider in this paper. The grammar in Figure 2 is based on the abbreviated syntax of XPath. In spite of its simplicity, the grammar in Figure 2 still captures all the intricate nuances.

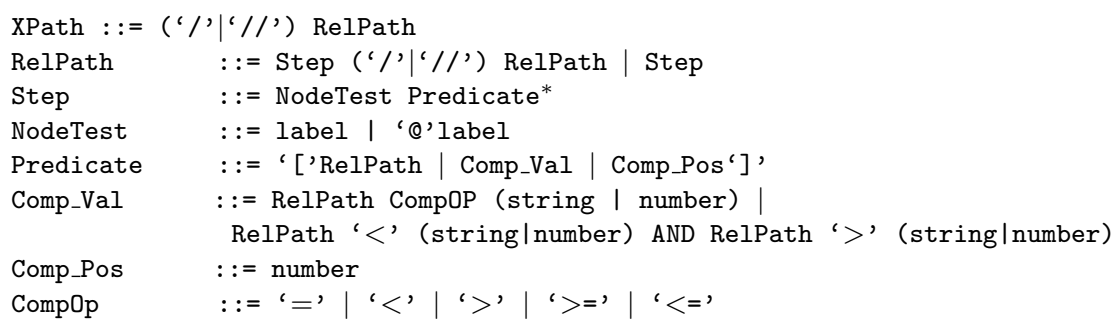

Fig. 2. A subset of XPath

Each step is connected by '/' and the default axis of a step is child which does not appear in the abbreviated syntax. '//' denotes the descendant axis ${ }^{1}$. Predicates act

${ }^{1}$ Strictly speaking, '//' is the abbreviated form of /descendant_or_self: $:$ node()/. However, by the presence of the default axis (i.e., child), // is interpreted as descendant, generally. 
as existence quantifiers. For example, //book[title] selects all book elements which have title elements as a child. Also, our subset of XPath supports value based predicates and the order based predicates in the forms of '[title= title1]' and '[2]', respectively.

To support an effective evaluation of path expressions, we devise a novel encoding method, called reverse arithmetic encoding, which is inspired by arithmetic encoding. We define some notations with a simple XML data to explain our proposed encoding method.

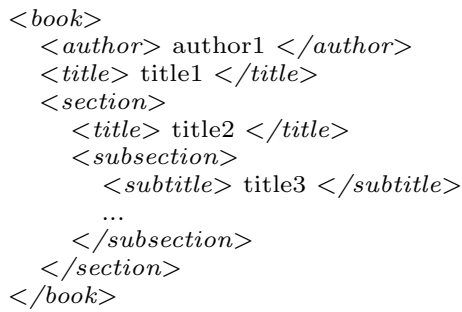

Fig. 3. An example of XML data

Definition 1. A simple path of an element $e_{n}$ in XML data is a sequence of one or more dot-separated tags $t_{1} \cdot t_{2} \ldots t_{n}$, such that there is a path of $n$ elements starting from the root element $e_{1}$ to $e_{n}$ and the tag of the element $e_{i}$ is $t_{i}$.

For example, in the XML data shown in Figure 3, the simple path of a subsection element is book.section.subsection.

Definition 2. When the simple path of an element $e$ in XML data is $a_{1} \cdot a_{2} \ldots a_{n}$, a dot-separated tag sequence $b_{k} \cdot b_{k+1} \ldots b_{n}$ is a label path of $e$ if we have $b_{k}=a_{k}$, $b_{k+1}=a_{k+1} \ldots b_{n}=a_{n}$, where $1 \leq k$ and $k \leq n$. Furthermore, for two label paths, $\mathrm{P}=p_{i} \ldots p_{n}$ and $\mathrm{Q}=p_{j} \ldots p_{n}$ of $e$, if $i \geq j$, then we call $\mathrm{P}$ is a suffix of $\mathrm{Q}$.

Again in Figure 3, section.subsection is a label path of the subsection element. And, subsection is a suffix of section.subsection. In XML, the structural constraints of queries are based on the label path such as //section/subsection.

Now, we present the reverse arithmetic encoding method. In contrast to existing XML compressors that transform the tag of each element to an identifier, reverse arithmetic encoding represents the simple path of an element by an interval of real numbers between 0.0 and 1.0. The basic idea of reverse arithmetic encoding is simple but elegant.

First, reverse arithmetic encoding partitions the entire interval $[0.0,1.0)$ into subintervals, one for each distinct element name as opposed to element node id. An interval for element $\mathrm{T}$ is represented as Interval $T$. The size of Interval $T$ is proportional to the frequency (normalized by the total frequency) of element $\mathrm{T}$. The following example shows the intervals for elements in Figure 3.

EXAMPLE 1. Suppose that the frequencies of elements $=\{$ book, author, title, section, subsection, subtitle $\}$ are $\{0.1,0.1,0.1,0.3,0.3,0.1\}$, respectively. Then, 
based on the cumulative frequency, the entire interval [0.0, 1.0) is partitioned as follows:

\begin{tabular}{c|c|c|c}
\hline element & frequency & $\begin{array}{c}\text { cumulative } \\
\text { frequency }\end{array}$ & Interval $_{T}$ \\
\hline book & 0.1 & 0.1 & {$[0.0,0.1)$} \\
author & 0.1 & 0.2 & {$[0.1,0.2)$} \\
title & 0.1 & 0.3 & {$[0.2,0.3)$} \\
section & 0.3 & 0.6 & {$[0.3,0.6)$} \\
subsection & 0.3 & 0.9 & {$[0.6,0.9)$} \\
subtitle & 0.1 & 1.0 & {$[0.9,1.0)$} \\
\hline
\end{tabular}

Next, reverse arithmetic encoding encodes the simple path $P=p_{1} \ldots \ldots p_{n}$ of an element $e$ into an interval $\left[\min _{e}, \max _{e}\right)$ using the algorithm in Figure 4.

Intuitively, the function reverse_arithmetic_encoding reduces Interval $p_{p_{n}}$ using the interval for the simple path $p_{1} \ldots p_{n-1}$ where $p_{n}$ is the tag of the element $e$. For understanding, we used a recursive function call in Line (4) of Figure 4. Basically, we encode the simple path of an element in a given XML data to an interval starting from the root element to other elements in the depth first tree traversal. Therefore, the recursion is not necessary in implementation since $\left[q_{\min }, q_{\max }\right)$ has already been computed at the time of encoding the parent element of $e$. Thus, the time complexity to compute all intervals of elements can be easily shown to be $\mathrm{O}(\mathrm{E})$, where $\mathrm{E}$ is the number of elements in a given XML data.

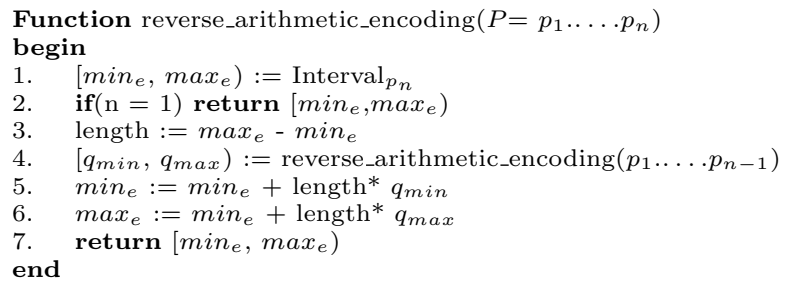

Fig. 4. An algorithm of reverse arithmetic encoding

Example 2 which is the continuation of Example 1 illustrates the behavior of reverse arithmetic encoding.

EXAMPLE 2. The interval $[0.69,0.699)$ for a simple path book.section.subsection in Figure 3 is obtained by the following process:

\begin{tabular}{c|c|c|c}
\hline element & simple path & Interval $_{T}$ & subinterval \\
\hline book & book & {$[0.0,0.1)$} & {$[0.0,0.1)$} \\
section & book.section & {$[0.3,0.6)$} & {$[0.3,0.33)$} \\
subsection & book.section.subsection & {$[0.6,0.9)$} & {$[0.69,0.699)$} \\
\hline
\end{tabular}

In the aspect of the utilization of the intervals, the reverse arithmetic encoding method is similar to the region numbering scheme originated in the field of information retrieval (IR) [Salminen and Tompa 1992]. Some XML storage systems [Li and Moon 2001] utilize the region numbering scheme to denote XML elements and attributes. The intervals (i.e., regions) generated by the region numbering scheme 
represent the relationship among elements (e.g., ancestor and descendant relationships). Suppose that there are two elements $\mathrm{x}$ and $\mathrm{y}$, where $\mathrm{x}$ is an ancestor of $\mathrm{y}$, then the corresponding intervals $\left(x_{s}, x_{e}\right)$ and $\left(y_{s}, y_{e}\right)$ for $\mathrm{x}$ and $\mathrm{y}$, respectively, satisfy the following property, that is $x_{s} \leq y_{s} \leq y_{e} \leq x_{e}$.

In contrast to the region numbering scheme, the intervals generated by reverse arithmetic encoding express the relationship among label paths as follows:

Property 1. Suppose that a simple path $P$ is represented as the interval $I$, then all intervals for suffixes of $P$ contain $I$.

For instance, the interval $[0.6,0.9)$ for a label path subsection and the interval $[0.69,0.78)$ for a label path section.subsection contain the interval $[0.69,0.699)$ for a simple path book.section.subsection. If a label path expression of a query is //section/subsection, this label path expression is represented as an interval $[0.69,0.78)$. And then, the query processor efficiently selects the elements whose corresponding intervals are within $[0.69,0.78)$. As a result, path expressions based on label paths are effectively evaluated by Property 1 .

Finally, without any loss of information, the start tag of an element $e$ is replaced by the minimum value of the subinterval generated by the function reverse_arithmetic_encoding. Since the minimum value of the subinterval is also consistent to Property 1, the corresponding tag of a minimum value can be obtained at the decompression phase easily using binary lookup of Interval $T_{T}$ s. In addition, path expressions are evaluated at the query processing phase effectively.

Furthermore, reverse arithmetic encoding can be naturally applied to some XML storage systems [Shimura et al. 1999; Tatarinov et al. 2002] which maintain the path information of individual elements by the path identifier.

Our encoding scheme belongs to the semi-adaptive compression. Since statistics, required in the XML compression phase, are collected and fixed at the preliminary scan, the generated code by XPRESS is independent to the location of the corresponding symbol (tags and data values).

If the adaptive compression such as adaptive huffman encoding is applied, the compression time is saved since the preliminary scan is not required. However, in the adaptive compression, the encoded value of a certain symbol is changed depending on the location of the occurrence of the symbol since the adaptive compression modifies the encoding model (e.g., huffman tree) dynamically. Thus, to evaluate a query with data value predicates, the complete decompression of compressed XML data is required. This degrades the query performance severely. Note that, generally, the XML data compression is an one time operation and queries are evaluated repeatedly. Therefore, the two-scan overhead on the XML data compression is compensated by frequent query evaluations.

Also, at the preliminary scan, XPRESS infers the type of data values of each distinct element. As described in Section 2, depending on the type of data values, the effective data encoding methods are different. However, existing XML compressors blindly use predefined encoding methods or apply some encoding methods manually. For example, in XMill, data values are bypassed to a built-in compression library, $z l i b$, if the data encoders are not specified manually. Additionally, in XGrind, the data values for elements and general attributes are compressed by huffman encoding and the data values of enumeration typed attributes are com- 
pressed by dictionary encoding. Without considering the nature of data values, the size of compressed XML data may increase. Therefore, we devise an effective type inference engine which infers the type of data values of each distinct element by simple inductive rules during the preliminary scan phase.

While, for numeric typed data values, XPRESS applies binary encoding first and then differential encoding with the minimum value. For example, data values of element $e$ "120", "150", "100" "130" are transformed into integers 120, 150, 100, 130 and encoded as 20,50, 0, 30. Since this encoding method preserves the order relationship among data values, the overhead of a partial decompression for numeric typed data is removed. For textual data, XPRESS adopts the arithmetic encoder and the dictionary encoder. As mentioned earlier, since the encoded values of arithmetic encoder preserve the order relationship among data values, the partial decompression is not required. But, the partial decompression for enumeration typed data is required. We apply the dictionary encoder when the number of distinct textual data is less than 128 . The compression ratio for dictionary encoding is high since encoded value consumes only one byte. Also, the original value of an encoded value is obtained efficiently using the hash table. Therefore, the partial decompression overhead for range queries on enumeration typed data values is relatively small.

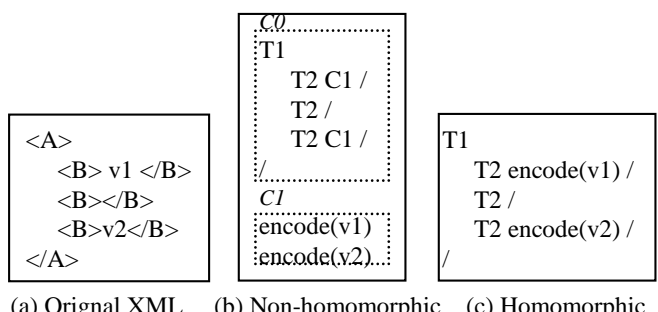

Fig. 5. An example of homomorphism

Like XGrind, XPRESS obeys the homomorphism [Tolani and Haritsa 2002]. The homomorphic compression technique preserves the structure of the original XML data on compressed XML data.

As shown in Figure 5-(b), some XML compression tools such as XMill physically separate structures (i.e., tag) and data (i.e., value). Here, the tags A and B are encoded as T1 and T2, respectively, and the end tags are replaced by '/'. By applying this technique, a built-in compression library such as zlib can reduce the size of compressed XML data well since the strings which have semantically/syntactically similar properties are grouped into a container. However, this technique incurs difficulty in query processing, also in performing the updates since the structure of compressed XML data is differentiated compared to the original XML data. In contrast to the non-homomorphic compression, since the homomorphic compression preserves the structure of the original XML data, the homomorphic compression allows us to evaluate queries and extract XML segmentations which satisfy given query conditions efficiently. Also, the homomorphic compression allows us to 
perform the updates efficiently since the updating XML segmentations are easily applied on compressed XML data.

As a result, based on the above features, the compressed XML data generated by XPRESS supports the query processing and the updates effectively without the complete decompression of compressed XML data.

\section{COMPRESSION TECHNIQUES IN XPRESS}

In this section, we present the architecture of XPRESS and detailed techniques developed for XPRESS.

Based on the features described in Section 3, we designed the architecture of XPRESS as depicted in Figure 6.

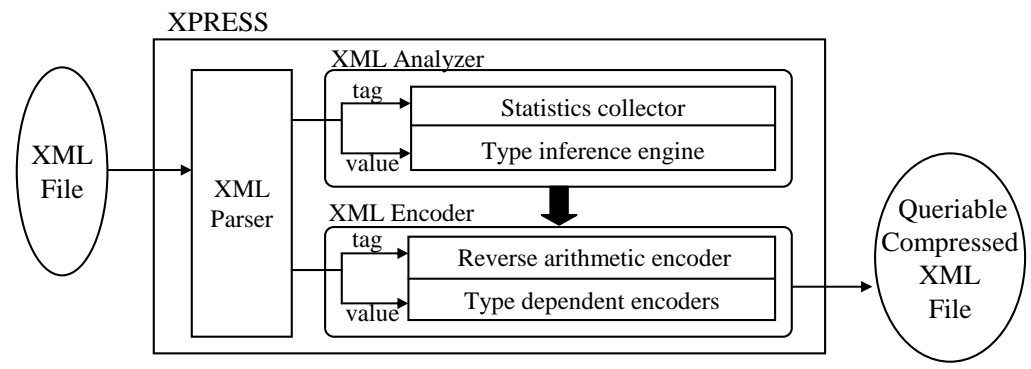

Fig. 6. The architecture of XPRESS

The core modules of XPRESS are XML Analyzer and XML Encoder. As mentioned earlier, the compression scheme of XPRESS is categorized as the semiadaptive compression. During the preliminary scan of given XML data, XML Analyzer (see details in Section 4.1) is invoked. XML Analyzer gathers the information used by XML Encoder (see details in Section 4.2) which generates queriable compressed XML data.

XML Analyzer consists of two submodules: the statistics collector and the type inference engine. The statistics collector computes the adjusted frequency (see Section 4.1) of each distinct element. The adjusted frequencies of elements are used as inputs to the reverse arithmetic encoder. The type inference engine infers the type of data values of each distinct element inductively and produces the statistics for the type dependent encoders in XML Encoder.

\subsection{XML Analyzer}

The main algorithm of XML Analyzer is shown in Figure 7.

To compute the frequency of each distinct element, the procedure Statistics_Collection is executed. To infer the types of data values, the procedure Type_Inferencing is executed. The algorithm XML_Analyzer generates a hash table called Elemhash. The entry of Elemhash is ELEMINFO which keeps the information (e.g., type of data values, frequency) of each distinct element. A stack called Pathstack is used to keep the trace of the currently visited element.

To get Interval ${ }_{T}$ for each distinct element, the statistics collector can simply count the number of occurrences of each distinct element. However, since tags of 


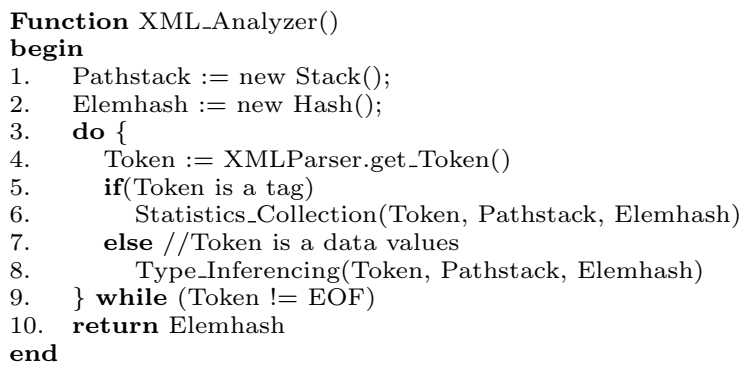

Fig. 7. An algorithm of XML Analyzer

higher level elements (e.g., the root element) appear rarely, the intervals for simple paths shrink quickly. This requires the use of high precision floating arithmetic.

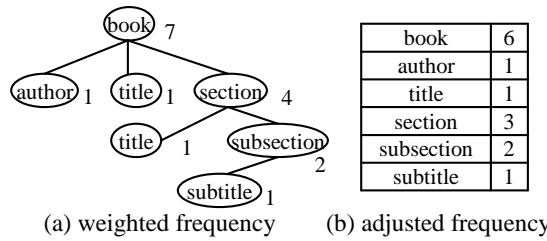

Fig. 8. Various frequencies

To prevent the rapid shrinking of an interval, we can use the concept of the path tree which is devised for the selectivity estimation of XML path expressions [Aboulnaga et al. 2001]. Every node in the path tree represents a simple path of XML data. The path tree of XML data in Figure 3 is shown in Figure 8-(a). In the original path tree of [Aboulnaga et al. 2001], each node keeps the number of elements reachable by the path starting from the root node to the node. As shown in Figure 8-(a), a node in our path tree keeps the number of subnodes including itself which we call the weighted frequency. Thus, intervals for higher level elements are enlarged and the intervals for simple paths do not shrink quickly. However, as mentioned in [Aboulnaga et al. 2001], the path tree consumes a large amount of memory, in the worst case, $\mathrm{O}(\mathrm{E}+\mathrm{T})$, where $\mathrm{E}$ is the number of elements and $\mathrm{T}$ is the number of distinct tags.

Thus, instead of using the path tree, we use a simple heuristic: if we visit an element whose tag is a new tag, then we increase the frequencies of elements which are ancestors of the currently visited element. Thus, like the path tree, the intervals for higher level elements are enlarged. We call this frequency the adjusted frequency.

Our simple heuristic method requires $\mathrm{O}(\mathrm{L}+\mathrm{T})$ space, where $\mathrm{L}$ is the length of the longest simple path in the given XML data and $\mathrm{T}$ is the number of distinct tags for the hash table which keeps the frequency of each tag. Furthermore, our method is more efficient than that of the path tree. Whenever a new node in the path tree is created, the weighted frequencies of ancestor nodes of the new node should be increased by 1 . However, our method increases the adjusted frequencies of ancestor nodes when an element with a new tag appears. As illustrated in Figure 8-(b), with 
the reduction of space requirement and enhanced performance, we can obtain the statistics similar to those of the path tree.

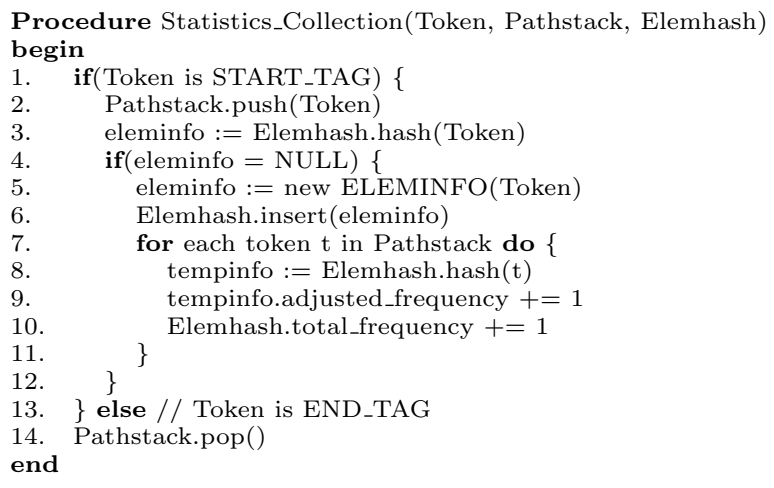

Fig. 9. The algorithm of the statistics collector

The algorithm of the statistics collector is presented in Figure 9. The input token is a tag. The trace of the currently visited element is kept by Pathstack (Line (2) and Line (14)). The hash at Line (3) is the hash function which returns an ELEMINFO for a given tag. Thus, when an element with a new tag appears, the hash function returns NULL (Line (4)). Then, the statistics collector makes an ELEMINFO for the element (Line (5)-(6)) and increases the adjusted frequencies for ancestors of the element including itself (Line (7)-(11)). At Line (10), we accumulate the total frequency to normalize the adjusted frequencies.

To produce the statistics of the inferred type for data values of each distinct element, the ELEMINFO has six fields: inferred_type, min, max, symhash, chars_frequency and Tag. The inferred_type field keeps the type of data values, up to now. The inferred_type is set as undefined initially. The min and max fields keep the track of the minimum binary value and the maximum binary value of data values, respectively. The symhash field is a hash table which keeps distinct data values. This symhash can be used as a dictionary for the dictionary encoder when the type of an element is the enumeration. The chars_frequency is an integer array which keeps the frequencies of individual characters of data values. This chars_frequency field is used to build intervals for the arithmetic encoder. The Tag field is used to keep the name of the element. To obtain the proper statistics of data values of each distinct element, the algorithm of the type inference engine shown in Figure 10 is executed.

The input token of Type_Inferencing is a data value. As mentioned above, Pathstack keeps the trace of currently visited elements. Thus, the tag of the element which is the owner of the given data value is at the top of Pathstack (Line (1) in Figure 10). Therefore, we obtain the corresponding ELEMINFO using this tag easily (Line (2)).

The function Infer_Type at Line (3) infers the type of the given data value using a simple rule as follows:

If all characters of the data value are numeric $\left({ }^{\prime} 0{ }^{\prime} \sim{ }^{\prime}{ }^{\prime}\right)$ and the first character is not ' 0 ', then Infer_Type returns integer which denotes that the data value is an 


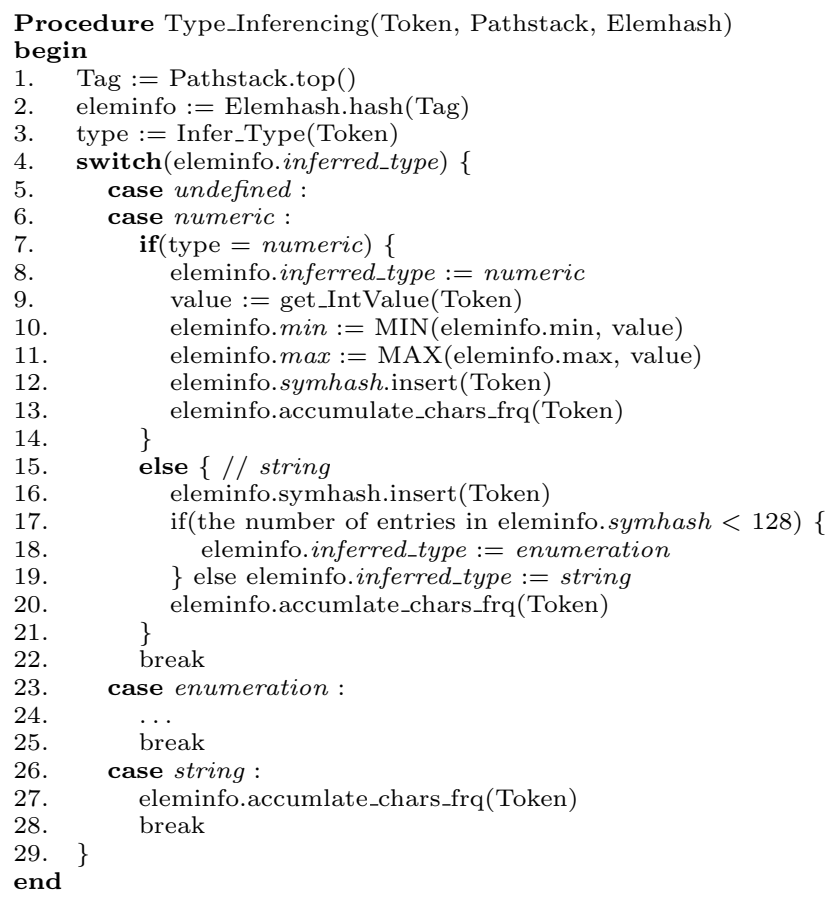

Fig. 10. The algorithm of the type inference engine

integer. If all characters of the data value are numeric (' 0 ' $\sim$ ' 9 ') with only one'.' and the first and second characters are ' 0 ' and '.' (i.e., in case of 0 .dddd), respectively, or the first character and the last character are not ' 0 ' nor '. ', respectively (i.e., in case of ddd.dddd), then a float is returned by Infer_Type. Otherwise, we consider the type of the data value as a string.

For brevity, integer and float types are represented as numeric type in Figure 10. However, the extension of the algorithm for integer type and floating type is straightforward.

If the type of the element is an numeric or undefined and the type of the given data value is an numeric (Line (5)-(14)), then we transform the data value into a binary value (Line (9)) and adjust the min and $\max$ fields using the binary value (Line (10)-(11)). The inferred type can be changed even though the currently inferred type is an numeric. Thus, to prepare for the future change, we also maintain the symhash field and chars_frequency field, properly (Line (12)-(13)).

If the type of the data value is a string (Line (15)-(21)), we change the inferred_type. Even though the preceding data values are numeric, we change the inferred_type since the numeric type does not express the string but the string type can express the numeric typed data using numeric characters. XPRESS has two types for textual data: enumeration and string. The string type is for general textual data, while the enumeration type is for the special string whose number of distinct values is less than 128 . To keep the distinct values, the hash table, symhash, discards duplicated strings (Line (12) and (16)). Thus, if the number of distinct 
entries of symhash is less than 128, we assign enumeration to the inferred_type. Otherwise, we assign string to the inferred_type. Also, because the inferred_type can be changed to string, the chars_frequency field is updated (Line (20)).

However, when the inferred_type is enumeration (Line (23)-(25)), we only check whether the inferred_type can be changed to string without considering the type of the given data value. Thus, Line (24) is the same as Line (16)-(20). If the inferred_type is string, the chars_frequency field is updated only (Line (26)-(28)).

\subsection{XML Encoder}

In this section, we describe the details of XML Encoder which compresses XML data using various encoders.

There are six encoders for data values in XPRESS, shown in Table I. Each distinct element has its own encoder which is one of six encoders.

\begin{tabular}{c|c}
\hline Encoder & Description \\
\hline $\mathbf{u 8}$ & encoder for integers where max-min $<2^{7}$ \\
$\mathbf{u 1 6}$ & encoder for integers where $2^{7}<=\max -\min <2^{15}$ \\
$\mathbf{u 3 2}$ & encoder for integers where $2^{15}<=$ max-min $<2^{31}$ \\
$\mathbf{f 3 2}$ & encoder for floating values \\
dict8 & dictionary encoder for enumeration typed data \\
arith & arithmetic encoder for textual data \\
\hline
\end{tabular}

Table I. Data Encoders

$\mathrm{u} 8, \mathrm{u} 16, \mathrm{u} 32$ and $\mathrm{f} 32$ are the differential encoders for numeric data and dict 8 and arith are the encoders for textual data.

As mentioned in Section 3, the encoders for numeric data transform the numeric data into binary and apply differential encoding with the minimum value obtained by the type inference engine. Note that the most significant bit (MSB) of the encoded value by the numeric data encoders is 0 . u 8 , u16 and u32 use 7 bits, 15 bits and 31 bits, and generate one byte, two bytes and four bytes, respectively.

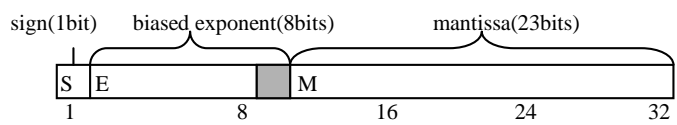

Fig. 11. IEEE 32bit floating point standard 754

A floating value generated by the encoder $\mathrm{f} 32$ is always positive since $\mathrm{f} 32$ generates the difference from the minimum value. Thus, the sign bit in Figure 11 is always 0 . Also, the encoder dict8 uses maximally 7 bits since, as described in Section 4.1, the number of distinct string values is less than $128\left(=2^{7}\right)$. Thus, the MSB of one byte generated by dict8 is also 0 .

In contrast to the other encoders, the encoder arith generates variable length encoded sequences. To parse this encoded sequence easily, we divide the encoded sequence into subsequences whose lengths are less than 128 and put one byte in front of each subsequence to denote the length of it. The encoded sequence whose length is less than 128 is not partitioned but has one byte for the length. Therefore, 
the MSB of each sequence or subsequence is always 0 since its length is less than 128. Consequently, in XPRESS, every MSB of encoded values for data values is 0 .

Until now, we described the encoders for data values. Next, we present the encoder for tags.

Start tags of individual elements are encoded by reverse arithmetic encoding using simple paths. In practice, we implement an approximated encoder, called the approximated reverse arithmetic encoder (ARAE), to improve the compression ratio and to parse compressed XML data without ambiguity.

Every MSB of the code generated by ARAE is 1. As mentioned above, every MSB of encoded data values is 0 . Thus, the parser for compressed XML data easily distinguishes data from structure.

To do this, ARAE adds 1.0 to the minimum floating value of the interval for a simple path. Since the minimum floating value generated by reverse arithmetic encoding is in $[0.0,1.0)$, the added value is in $[1.0,2.0)$. According to the IEEE floating point representation (see Figure 11), a floating value is represented as $\mathrm{S} \times 1 . \mathrm{M} \times 2^{E-127}$. For example, the binary representation of 1.25 is 1.01 and this is transformed into $1 \times 1.01 \times 2^{0}$. Thus, $\mathrm{S}=0, \mathrm{E}=0+127=01111111$, and $\mathrm{M}$ $=[1]$.01 . The first bit $^{2}$ of the second byte for every floating value in $[1.0,2.0)$ is always 1 since the sign bit and the biased exponent are 0 and 127 (=0111 1111), respectively. Thus, by cutting the first byte, the MSB of the code generated by ARAE is always 1.

In addition, to reduce the size of compressed XML data, ARAE truncates the last byte. Due to the reduction of the precision, the code generated by ARAE may not always represent the corresponding simple path exactly. However, at least, the code generated by ARAE represents the tag of an element. As described in Example 3, the generated code still represents a label path (i.e., a suffix of a simple path).

Example 3. Suppose that ARAE truncates digits less than $10^{-2}$ (i.e., last 17 bits) and that tags and corresponding Interval $_{T} s$ are the same as those in Example 1.

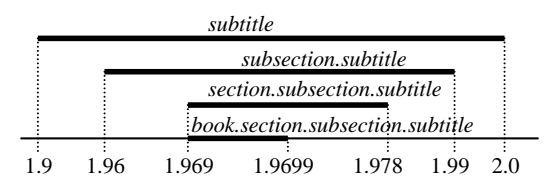

The interval for a simple path book.section. subsection.subtitle is $[1.0+0.9+$ $0.1 \times 0.69=1.969,1.0+0.9+0.1 \times 0.699=1.9699)$. Then, the truncated value is 1.96 which is in the interval $[1.96,1.99)$ for subsection.subtitle.

Therefore, this approximation does not damage the accuracy and the efficiency of query processing. Recall that the reduction of data size by the data compression induces the performance improvement due to the reduction of disk I/Os. Furthermore, common structural constraints of XML queries are partial matching path expressions based on label paths instead of simple paths since users may not know or may not be concerned with the detailed structure of XML data and intentionally

${ }_{2 \text { it is represented by the gray box in Figure } 11}$ 
make the partial matching path expression to get intended results. But, note that too much approximation incurs the inefficiency of query processing since a label path represented by the encoded value becomes too short.

Finally, to distinguish start tags and end tags, the interval $[1.0+0.0=0 \mathrm{x} 8000$, $\left.1.0+2^{-7}=0 \mathrm{x} 8100\right)$ is reserved. For all end tags, one byte $0 \times 80(=10000000)$ is assigned since the codes for the interval start with $0 \mathrm{x} 80$. And codes for start tags are always greater than or equal to $0 \times 8100$. Therefore, the parser for compressed XML data distinguishes the codes for start tags and the codes for end tags.

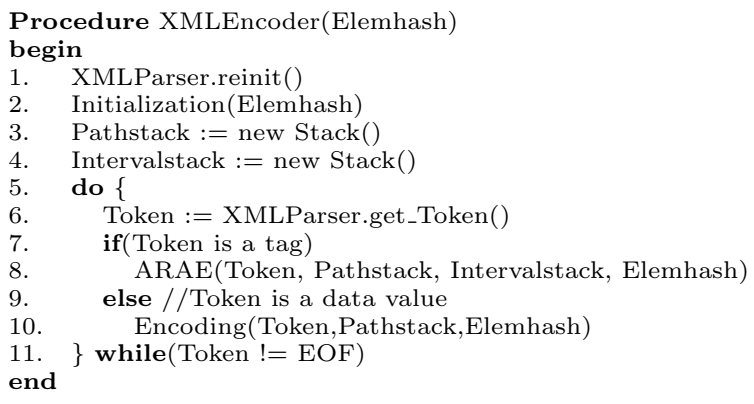

Fig. 12. The algorithm of XML Encoder

The algorithm of XML Encoder is in Figure 12. First, XMLParser is reinitialized to rescan a given XML file (Line (1)). Then, for each distinct element, XML Encoder calculates Interval ${ }_{T}$ and chooses a proper encoding method (e.g., u8) using the function Initialization (Line (2)). To compute Interval ${ }_{T}$, we used the interval $\left[2^{-7}, 1.0-2^{-15}\right]$ as the entire interval instead of $[0.0,1.0)$ since $\left[0.0,2^{-7}\right)$ is reserved for end tags and the value less than $2^{-15}$ can not be represented using 15 bits. Also, for the same reason, we adjusted the length of Interval ${ }_{T}$ to a number greater than $2^{-15}$. In general, this case does not appear.

Pathstack is used to keep the information of an owner element of data values (Line (3)). To compute the interval for the currently visited element, the interval for the parent element is required. To keep the interval for a parent element, a stack, called Intervalstack, is created (Line (4)). And then, the token generated by XMLParser is compressed by encoders of XPRESS (Line (5)-(11)).

\subsection{Query Processing}

To evaluate queries on compressed XML data generated by XPRESS, we devise a query processor.

XPRESS is a homomorphic compressor and the MSB of encoded tag (i.e., the reverse arithmetic encoding value) is 1 , while the MSB of encoded data values is 0 . The behavior of query executor is similar to the query executor using SAX parser (an XML parser). In contrast to the general SAX parser, for processing path expressions, XPRESS maintains encoded tag values compressed by the reverse arithmetic encoding which enables an efficient processing of path expressions. Also, XPRESS maintains encoded data values, instead of strings, so that value comparisons are 
efficiently performed. Therefore, we only present details of our query executor with respect to the reverse arithmetic encoding and value comparison. The query processor consists of a query parser, a query transformer, and a query executor.

The query parser separates the values from the label path expression when the query contains any value comparison. Also, to support complicated partial matching path queries whose examples are provided in Table III of Section 6.1, the query parser breaks down a complicated path expression into multiple single path expressions $^{3}$ according to the occurrence of ' $/ /$ '.

For example, a complicated path expression $P=/ / p_{1} / p_{2} / p_{3} / / p_{4} / p_{5} / p_{6}$ is partitioned into $P_{1}$ and $P_{2}$, where $P_{1}=/ / p_{1} / p_{2} / p_{3}$ and $P_{2}=/ / p_{4} / p_{5} / p_{6}$. The query executor looks for the elements which satisfy $P_{1}$ and evaluates $P_{2}$ among the descendants of $P_{1}$ 's results.

The query transformer transforms the single path expressions to intervals. First, the query transformer partitions each long single path expression obtained by the query parser into short single path expressions whose corresponding interval sizes are greater than $1.0+2^{-15}$ since an interval for each element is expressed based on the precision of $1.0+2^{-15}$, as mentioned in Section 4.2. Suppose that a single path expression $P_{s}=/ / p_{1} / \ldots / p_{n}$ requires a precision higher than $1.0+2^{-15}$ and $P_{s}^{\prime}=/ p_{1} / \ldots / p_{i}$ requires a precision lower than $1.0+2^{-15}$. Then, $P_{s}$ is partitioned as $P_{s}=P_{s}^{\prime} P_{s}^{\prime \prime}$, where $P_{s}^{\prime \prime}=/ p_{i+1} / \ldots / p_{n}$. Also, if a precision higher than $1.0+2^{-15}$ is required for $P_{s}^{\prime \prime}$, we apply this partitioning process repeatedly. Thus, the query transformer transforms the partitioned single path expression into a sequence of intervals.

Finally, by using the sequence of intervals transformed by the query transformer, the query executor evaluates the tokens of encoded elements in compressed XML data whether their encoded values are in an interval of the sequence or not.

In the original XML document, the data value is a textual string. Thus, to evaluate value-based predicates, a string comparison is required in uncompressed XML data. In contrast, various comparison operators according to the type of data values are applied in XPRESS. The type of data value with owner element's tag $T$ is obtained easily using binary lookup of Interval $_{T}$. Therefore, we reduce the overhead of the decompression and comparison operators.

For the exact matching query, a data value of exact matching conditions in a query is converted into an encoded value using the type dependent encoder as described in Section 4.2. Then, the query executor detects the elements which satisfy the label path expression and the value comparison without decompression.

For the range query, the range condition for a numeric typed element is encoded by the type dependent encoder for the element. Then, without the decompression of encoded values, the query is evaluated since the type dependent encoders for numeric typed elements preserve the order information among data values.

As mentioned earlier, XPRESS applies the dictionary encoder and the arithmetic encoder to textual data. For the range condition of a textual typed element, a partial decompression is required when the data value is a enumeration type since the dictionary encoder (i.e., dict8) does not preserve the order information among data values. However, since the original data value is efficiently obtained by the

\footnotetext{
${ }^{3}$ A single path expression denotes a path expression that contains at most one occurrence of '//'.
} 
hash table, the partial decompression overhead is relatively small.

In the preliminary version of XPRESS, the general string values are encoded by the huffman encoding method. Since the encoded values generated by huffman encoder do not preserve the order information of original values, the partial decompression is required for range queries. In contrast to the huffman encoder, the arithmetic encoder preserves the order information among data values. Thus, a partial decompression is not required when the data value is a string type.

In addition, for order based predicates, the order among sibling elements is easily computed since XPRESS preserves the structure of the original XML data.

\section{UPDATE PROCESSING}

In this section, we present the details of the update processor which supports direct updates on compressed XML data.

The naive approach for updates on compressed XML data is that original XML data is constructed by the complete decompression and the update and the recompression are performed on uncompressed XML data. In this case, the system resource and time are wasted. Thus, we devised an update processor for compressed XML data which does not perform a complete decompression.

Basically, the update operations can be categorized by three types: deletion, insertion, and change. The deletion is trivially performed by eliminating a certain portion of compressed XML data specified by the query. The change is considered as the combination of the deletion and the insertion. Thus, in this paper, we only focus on the insertion on compressed XML data.

Our subset of XPath queries contains the order based predicate and value based predicate. By using the predicates, XPRESS specifies the insertion (or deletion) points on the compressed XML data.

The other XML compressors do not consider direct updates on compressed XML data. Basically, since XPRESS is a homomorphic and queriable compressor, direct updates on the compressed XML data is possible. Similarly, XGrind can also support direct updates. However, XGrind does not consider XML updates on compressed XML data.

As mentioned earlier, XPRESS encodes tags and data values according to the statistics (e.g, frequencies of tags, types of data values).

Actually, inserting and deleting XML elements that appeared in the original XML document affect the frequencies of tags. However, to reduce the update cost, the statistics for reverse arithmetic encoding are only renewed when new tags appear in updates. This approach still guarantees correct query processing since the reverse arithmetic encoding values obtained by reusing the statistics satisfy Property 1 . In order to maintain the correct statistics, it is necessary to recompress compressed XML documents periodically.

The newly inserted XML fragment may affect currently compressed XML data. Thus, by analyzing the newly inserted XML fragment, the update processor of XPRESS renews the statistics. And then, with respect to the difference between the renewed statistics and the current statistics, a partial decompression of compressed XML data is performed. Using the renewed statistics, the XML fragment and the partially decompressed XML data are recompressed.

ACM Transactions on Internet Technology, Vol. V, No. N, Month 20 YY. 


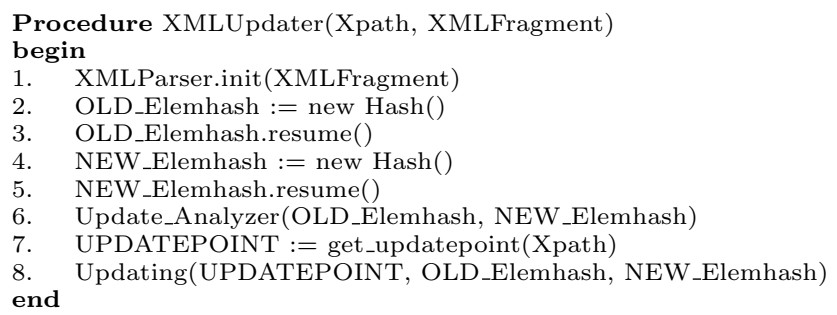

Fig. 13. The algorithm of XML Updater

The main algorithm for the update processor of XPRESS is depicted in Figure 13. Two input parameters of XMLUpdater are Xpath and XMLFragment. Xpath is an XPath query which is used to specify the insertion points on the compressed XML data. And, XMLFragment is the newly inserted XML fragment.

First, to parse XML fragment, XMLParser is initialized with the given XML fragment (Line (1)). At Line (2)-(5), the algorithm XMLUpdater generates two hash tables: OLD_Elemhash and NEW_Elemhash. OLD_Elemhash is used to keep the current statistics and NEW_Elemhash is used to keep the renewed statistics. By invoking the method resume, the statistics are reloaded into each hash table from the header of compressed XML data. At the beginning, OLD_Elemhash and NEW_Elemhash are the same, and NEW_Elemhash is changed subsequently.

The core modules of XMLUpdater are Update Analyzer and Updating. Similar to the compression scheme of XPRESS that is categorized as the semi-adaptive compression, XMLUpdater of XPRESS requires two scans of XML fragment. The procedure Update_Analyzer (see details in Section 5.1) is invoked to renew the statistics (Line (6)).

By using the query processor of XPRESS, the get_updatepoint function at Line (7) finds an UPDATEPOINT where the XML fragment is required to be inserted. For brevity, in Figure 13, we assume that there is only one UPDATEPOINT on given compressed XML data. However, the extension to maintain multiple UPDATEPOINTs is straightforward.

The procedure Updating (see details in Section 5.2) is invoked to insert the XML fragment at the UPDATEPOINT and re-encode certain portions of compressed XML data using the statistics gathered by the procedure Update_Analyzer (Line $(8))$.

\subsection{Update Analyzer}

The algorithm of Update Analyzer is presented in Figure 14. Basically, Update Analyzer renews the statistics by scanning the XML fragment.

Pathstack is used to identify the tag of owner element of a data value in the XML fragment.

The newly inserted XML fragment may incur two kinds of violations with respect to the current statistics kept in OLD_Elemhash. The first is the appearance of new tags. The second is the change of the inferred data type.

As described in Section 3, the reverse arithmetic encoder for tags partitions the entire interval $[0,1)$ into subintervals, one for each distinct tag. Thus, the intervals 


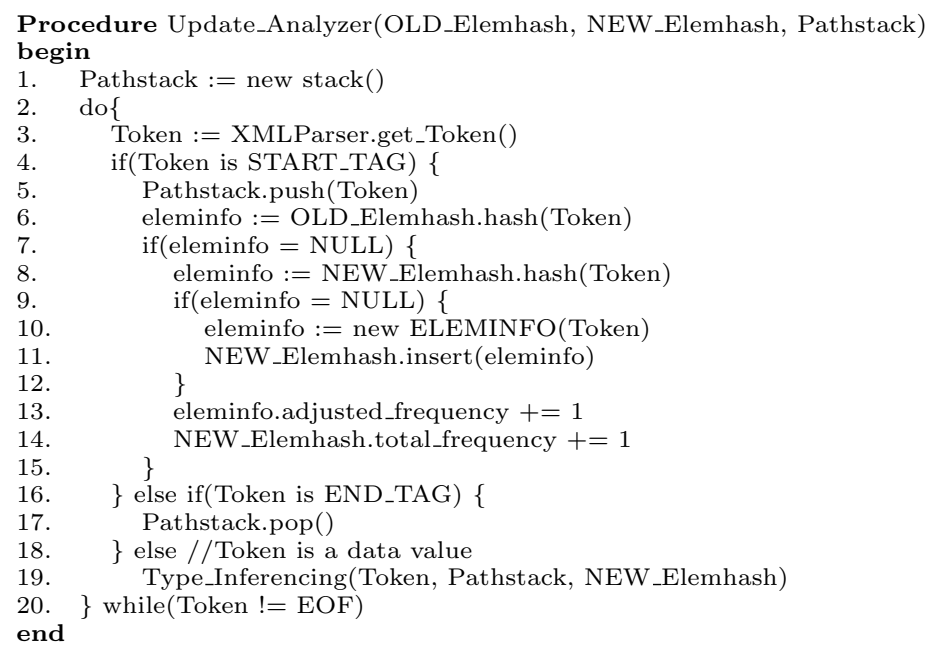

Fig. 14. The algorithm of Update Analyzer

for new tags do not exist in the current statistics which are kept in OLD_Elemhash. Thus, to assign the intervals to new tags, the frequency of new tags is computed. When an element with a new tag appears (Line (7)-(15)), the hash function of OLD_Elemhash returns NULL since the tag has not appeared on compressed XML data before (Line (7)). Also, if this tag appears for the first time, the hash function of NEW_Elemhash returns NULL. In this case, the procedure Update_Analyzer makes an ELEMINFO for the element and inserts it into NEW_Elemhash (Line (9)-(12)). Then, Update_Analyzer increases the adjusted frequency of the element and the total frequency of NEW_Elemhash by 1 (Line (13)-(14)).

Lastly, for data values in the XML fragment, the algorithm of the type inference engine described in Figure 10 is invoked with NEW_Elemhash (Line (19)). Thus, by the comparison between type information kept in OLD_Elemhash and type information kept in NEW_Elemhash, we can identity the change of the data type.

\subsection{Updating}

The Updating module inserts the XML fragment into UPDATEPOINT on compressed XML data and changes portions of compressed XML data which are affected by the XML fragment. The algorithm of Updating is shown in Figure 15.

At the beginning of the algorithm Updating, the initialization for NEW_Elemhash is performed to calculate Interval $T$ and choose a proper encoding method as described in Section 4.2 (Line (1)).

For the initialization of OLD_Elemhash, choosing proper decoding methods is additionally required since the partial decompression of compressed data values is necessary if the inferred_type is changed to another type (Line (2)).

NEW_Codestack is used to keep the trace of currently visiting element by using the encoded value for the tag (Line (3)). In this case, if new tags appear in the XML fragment, NEW_Codestack is maintained with the newly calculated intervals 


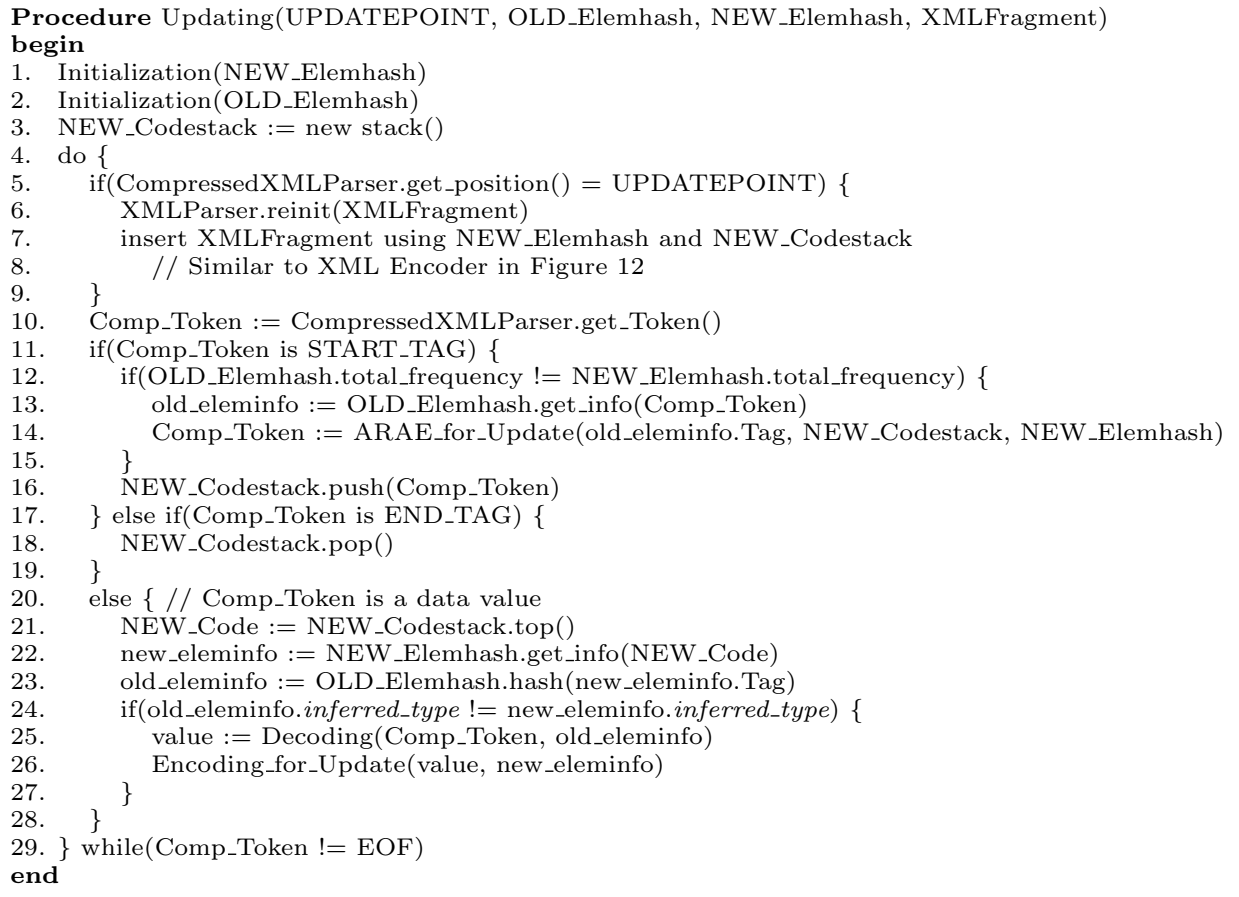

Fig. 15. The algorithm of Updating

from NEW_Elemhash after invoking Update_Analyzer.

As mentioned previously, our proposed update processor directly inserts the XML fragment into compressed XML data. Thus, to parse compressed XML data, a specific parser called CompressedXMLParser is used. CompressedXMLParser generates a Comp_Token while traversing compressed XML data. As mentioned in Section 4, classifying Comp_Tokens as START_TAG, END_TAG, and data values is easy since all the MSBs of the encoded values for tags start with 1 while all the MSBs for data values are 0. Especially, for END_TAGs, XPRESS assigned 0x80 $(=1000$ 0000).

When the parsing position of CompressedXMLParser is the same as UPDATEPOINT, the XML fragment is inserted (Line (5)-(9)). First, XMLParser is reinitialized for the XML fragment (Line (6)). Next, as commented at Line (8), the XML fragment is compressed by using NEW_Elemhash and NEW_Codestack, and then inserted. This process is similar to XML Encoder described in Figure 12.

As described in Section 5.1, the total frequency of NEW_Elemhash is increased only when a new tag appears in the XML fragment. Thus, if the XML fragment has new tags, the total frequency of OLD_Elemhash and the total frequency of NEW_Elemhash are different.

For each START_TAG, if the two total frequencies are different, a new encoded value for each tag is required since the subinterval, Interval ${ }_{T}$, for each tag is changed (Line (12)-(15)). Otherwise, Comp_Token is simply pushed into NEW_Codestack (Line (16)). 
Note that the value of Comp_Token is the minimum value of the interval generated by the reverse arithmetic encoder using OLD_Elemhash. By checking the containment relationship between the minimum value (=Comp_Token) and each Interval $_{T}$ in OLD_Elemhash, proper element information (i.e., ELEMINFO) can be obtained. Thus, the procedure Updating obtains the related ELEMINFO, old_eleminfo, by calling OLD_Elemhash.get_info (Line (13)).

From old_eleminfo, the procedure Updating gets the tag (= old_eleminfo.Tag) of currently visiting element and the new encoded value of the parent element's tag which is kept at the top of NEW_Codestack. Thus, by invoking ARAE_for_Update, the new encoded value of Comp_Token is computed and replaced (Line (14)).

Lastly, for data values, the algorithm Updating obtains an ELEMINFO from OLD_Elemhash, and an ELEMINFO from NEW_Elemhash (Line (21)-(23)). The encoded value of the tag of the element which is the owner of the given compressed data value is at the top of NEW_Codestack. Thus, by calling NEW_Elemhash.get_info with the encoded value of the owner element's tag, the new_eleminfo is obtained. And, using new_eleminfo.Tag, the old_eleminfo is acquired from OLD_Elemhash.

Then, their inferred_types are compared at Line (24). Thus, if their inferred_types are different, the decompression of the compressed data value (i.e., Comp_Token) and the recompression of the decompressed value are performed (Line (25)-(26)).

Note that, at Line (24), much more precise comparison is required since the min or max can be changed even though the inferred_type is not changed. In Figure 15, we did not show the comparisons for such cases for brevity. However, an extension to handle such cases is straightforward.

\section{EXPERIMENTS}

To show the effectiveness of XPRESS, we empirically compared the performance of XPRESS with two representative XML compressors XMill ${ }^{4}$ and XGrind $^{5}$ as well as a general compressor, gzip, using real-life XML data sets. In our experiments, XPRESS shows a reasonable compression ratio compared to XMill. We compared the query performance of XPRESS to that of XGrind. In addition, to show the effectiveness of the queriable XML compressor, we compared the query performance of XPRESS with that of XMill. Since XMill does not support direct querying on compressed XML data, we implemented a query engine for uncompressed textual XML data, based on the algorithms used in the query processor of XPRESS. The query engine for XMill is a modified version of the query engine from XPRESS. The query engine for XMill handles uncompressed textual XML data whereas the version from XPRESS handles compressed XML data. Therefore, the query engine for XMill keeps the trace of tags to compute the path expression. For XMill, another alternative is to feed the SAX events generated by the XMill decompressor directly into the XPath processor. While this alternative eliminates the need for re-parsing for query processing after parsing in the process of decompression, the alternative also incurs the complete decompression and requires recompiling the XPath processor. Therefore, in our experiment, we decompressed the compressed XML data generated by XMill, and executed the query engine on uncompressed XML data.

\footnotetext{
4available in http://www.research.att.com/sw/tools/xmill/

5 available in http://sourceforge.net/projects/xgrind/

ACM Transactions on Internet Technology, Vol. V, No. N, Month 20YY.
} 
For XGrind, we also implemented a new query processor since the original query processor of XGrind does not support predicates and partial matching path expressions. Lastly, since there is no XML compressor which supports direct updates on compressed XML data, for evaluating the efficiency of the update processor of XPRESS, we compared the update performance of XPRESS with that of a naive approach using XMill. As mentioned in Section 5, the naive approach decompresses compressed XML data, updates decompressed XML data, and finally, recompresses updated XML data. XPRESS shows significantly better query performance than XGrind and update performance than the naive approach using XMill.

\subsection{Experimental Environment}

The experiments are performed on a Sun Ultra Sparc II $168 \mathrm{MHz}$ platform with Solaris 2.5.1 and 384 MBytes of main memory. The data sets are stored on a local disk. In our experiments, XMill does not have any user-specified encoders.

Data Sets We evaluated XPRESS using a number of real-life XML data sets: Univ, MapData, Part, Lineitem, Orders, SigmodRecord, Baseball, Shakespeare, SwissProt, and DBLP. The characteristics of the data sets used in our experiment are summarized in Table II. Size denotes the disk space of XML data in MBytes, Depth is the length of the longest simple path of each XML data set, Tags indicate the number of distinct tags, Numeric represents the number of distinct elements whose data values' type is numeric (i.e., integer or float), and Enum indicates the number of distinct elements whose data values' type is enumeration.

\begin{tabular}{c|c|c|c|c|c}
\hline Data Set & Size(MB) & Depth & Tags & Numeric & Enum \\
\hline Univ & 2.33 & 5 & 22 & 0 & 9 \\
MapData & 5.97 & 6 & 9 & 1 & 1 \\
Part & 0.62 & 3 & 12 & 3 & 6 \\
Lineitem & 32.29 & 3 & 19 & 8 & 5 \\
Orders & 5.37 & 4 & 12 & 4 & 3 \\
SigmodRecord & 0.22 & 7 & 12 & 4 & 1 \\
Baseball & 1.06 & 6 & 46 & 32 & 5 \\
Shakespeare & 7.64 & 6 & 21 & 0 & 5 \\
SwissProt & 114.82 & 5 & 99 & 3 & 6 \\
DBLP & 133.85 & 6 & 41 & 2 & 10 \\
\hline
\end{tabular}

Table II. XML Data Set

The Univ [UW ] address the description of courses held in Universities. Since they are for the description of courses, they have some integer values to indicate credits, and class rooms, as well as some enumerated values to describe course code, title, days of classes, and building names.

The MapData [ETRI ] is an instance of GML [Open GIS Consortium ] which is an XML specification for the geographical application. Since the MapData contains the geographical information for a region of Seoul (the capital city of South Korea), a large number of floating values for coordinates exists.

Part, Lineitem, and Orders [UW ] are the XML versions of TPC-H benchmark data used widely in the field of relational databases. They have several numeric typed elements to describe the key values.

The SigmodRecord [UW ] provides an index of articles from SIGMOD Record. Since it describes information related to the articles, most elements are string typed 
except a small number of numeric typed elements (i.e., volume numbers, page numbers).

The Baseball [Harold] contains the complete baseball statistics of all players of each team that participated in the 1998 Major League. Since it contains statistics, it has many numeric typed elements.

The Shakespeare [Cover 2001] is the collection of plays of Shakespeare which is marked up by Jon Bosak. Since it describes the overall scenario of plays of Shakespeare, the Shakespeare does not have any numeric typed elements.

The SwissProt [UW ] is a protein sequence database which provides a high level of annotations such as the description of the function of a protein, its domain structure, post-translational modifications, and variants. Due to a large amount of descriptions, string typed elements are dominant.

The DBLP(Digital Bibliography Library Project) [UW ] provides bibliographic information on major computer science journals and proceedings. Since it describes general information related to journals and proceedings, most of elements are string typed, similar to the SwissProt.

\begin{tabular}{c|c}
\hline Type & Query Example \\
\hline 1 & /root/course/time/start_time \\
2 & //course/place//building \\
3 & //course[5]/place/building \\
4 & //course/place[building = "ELIOT"] \\
5 & $/ /$ course/place[building $>$ "CHEM" and building $<$ "SPORTS"] \\
6 & /root//course/place[building = "ELIOT"]/room[1] \\
\hline \multicolumn{2}{c}{ Table III. XML Query Examples }
\end{tabular}

Queries We evaluated XPRESS using several queries. The characteristics of queries used in our experiment are described in Table III.

The number in the first column represents the type of queries. The queries of type 1 are path expressions based on the simple path, the queries of type 2 are partial matching path expressions, the queries of type 3 are complicated partial matching path expressions with order based predicates, the queries of type 4 and 5 are complicated partial matching path queries with the exact data value and the range of data values, respectively, and the queries of type 6 are the branch queries with predicates. Query Example in Table III shows the examples of corresponding XPath queries.

We choose these kinds queries for the following reasons. Queries of type 1 evaluate the query performance for long path expressions. Queries of type 2 test the query performance of simple partial matching path queries. Query type 3 is similar to query type 2 but contains order based predicates. To measure the query performance of value based predicates, we chose Query type 4 and 5. Finally, to measure the query performance of branch queries, we choose Query type 6. Query type 6 represents the most complicated queries.

Update Queries We evaluated the update processor of XPRESS using several updates. The characteristics of updates are described in Table IV.

The number in the first column is used to denote the type. In Table IV, the updates of type 1 are the insertions of XML fragments whose tags already appeared and the types of data values are not changed, the updates of type 2 are the insertions 


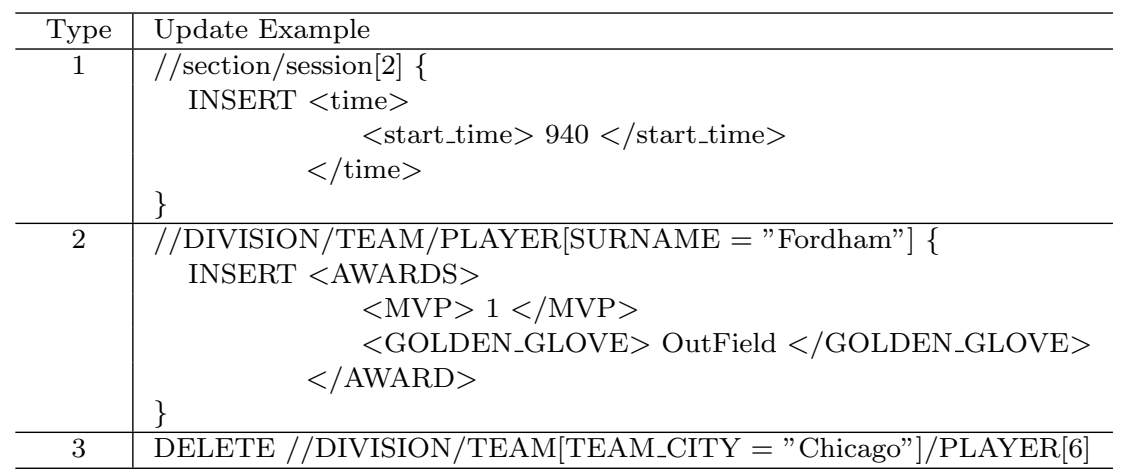

Table IV. XML Update Query Examples

of XML fragment whose tags newly appeared, and the updates of type 3 are the deletions on the compressed XML data. Update Example in Table IV shows the examples of corresponding update queries represented using the syntax introduced in [Tatarinov et al. 2001].

\subsection{Experimental Results}

In this section, we first present the compression ratio of each compressor. The compression ratio is defined as follows:

$$
\text { Compression ratio }=1-\frac{\text { Size of compressed XML data }}{\text { Size of original XML data }}
$$

Then, we report the compression time of each compressor. In addition, to show the effect of zlib in XMill, we show the compression ratio of gzip which is applied to the compressed XML data of XPRESS and XGrind. Then, we show the query performance of XPRESS with that of XGrind and the query engine for XMill. The update performance of XPRESS with that of the naive approach using XMill is presented last.

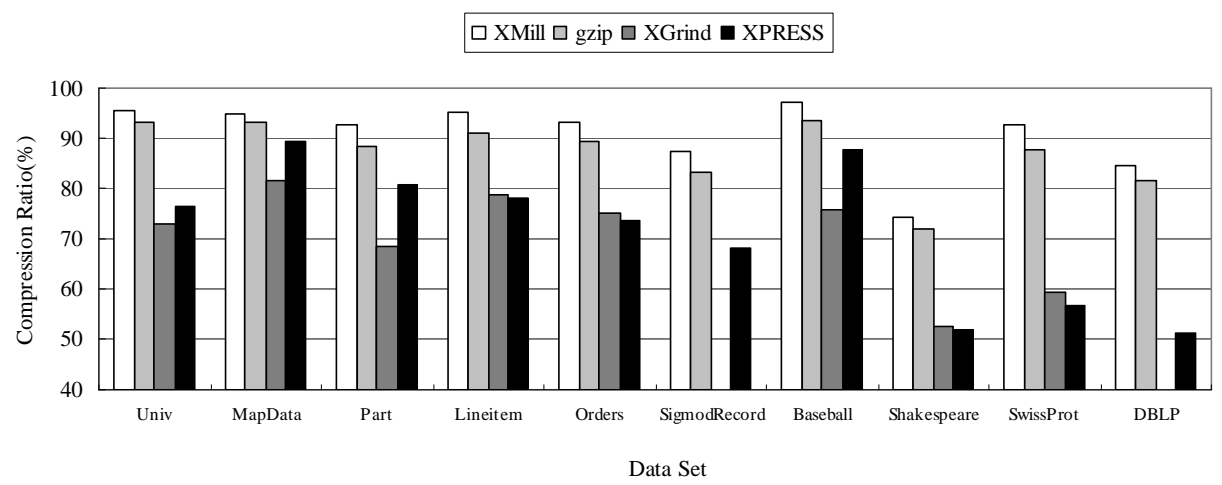

Fig. 16. Compression ratio 
Figure 16 shows the compression ratios for different data sets and compressors. For each different size of XML data set, the four connected bars represent XMill, gzip, XGrind, and XPRESS. Since XMill uses the dictionary encoding method for structural information, and groups semantically related data values into containers before compressing with zlib, as we expected, XMill achieved the best compression ratio, on the average of $90 \%$. The average compression ratio of XPRESS is $71 \%$. The compression ratio of XPRESS depends on the characteristics of data values, not on the sizes of XML data. Since XPRESS uses the type inference engine to apply appropriate compression methods for data values, it performs well if the data values are enumeration, floating, or integer type. Thus, the compression ratio of XPRESS for the Baseball and the MapData is better than that for the other data sets. As shown in Table II, since the Shakespeare and the SwissProt do not have much numeric and enumeration typed data, the compression ratio of XPRESS is just slightly lower than that of XGrind. In our experiment, XGrind does not compress the SigmodRecord and the DBLP data sets. We can observe that XPRESS shows a reasonable compression ratio for all cases.

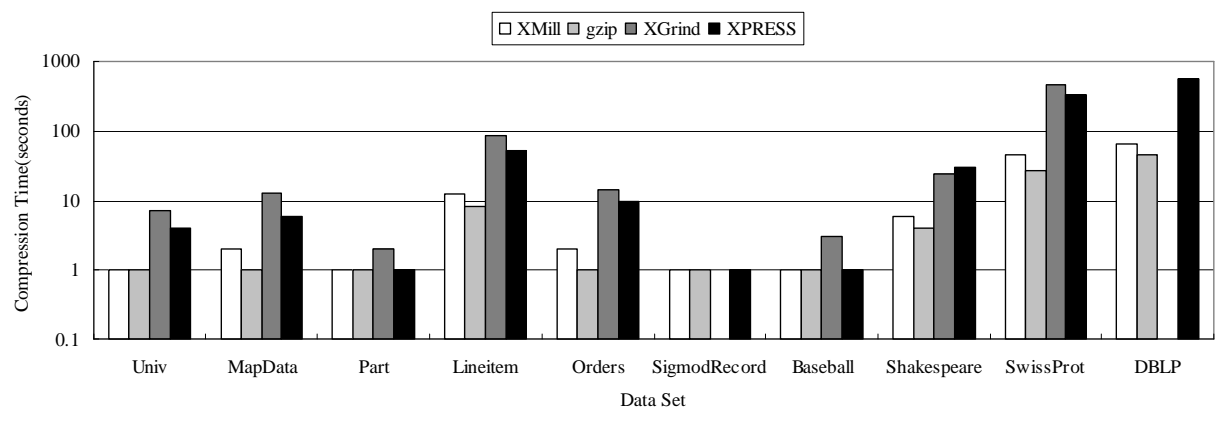

Fig. 17. Compression time (log scale)

Figure 17 shows the compression time of each compressor based on the log scale ${ }^{6}$. In our experiments, XGrind generally shows the worst compression time. As mentioned earlier, to determine the data value encoders (i.e., huffman encoding and dictionary encoding), XGrind uses DTDs. To parse and obtain some information from DTDs, XGrind adopts a shareware XML parser. Thus, the overhead of XML parsing and DTD validation is significant. In contrast to XGrind, XPRESS and XMill parse the XML document efficiently since they do not use any information from DTDs.

As mentioned earlier, XGrind does not compress the SigmodRecord and the DBLP data sets. Thus, we did not report the compression time of XGrind for these data sets. XMill and gzip show the best performance of data compression since they compress XML data by one scan. Using proper encoding methods that are determined by the inferred types, XPRESS shows better compression time

\footnotetext{
${ }^{6}$ some bars for compression time of some data sets are not shown in the graph since their values are less than or equal to 1 .
}

ACM Transactions on Internet Technology, Vol. V, No. N, Month 20YY. 
compared to that of XGrind. In the evaluation of the decompression time, the result shows a similar pattern compared to that of the compression time. Thus, we omit the graph of the decompression time.

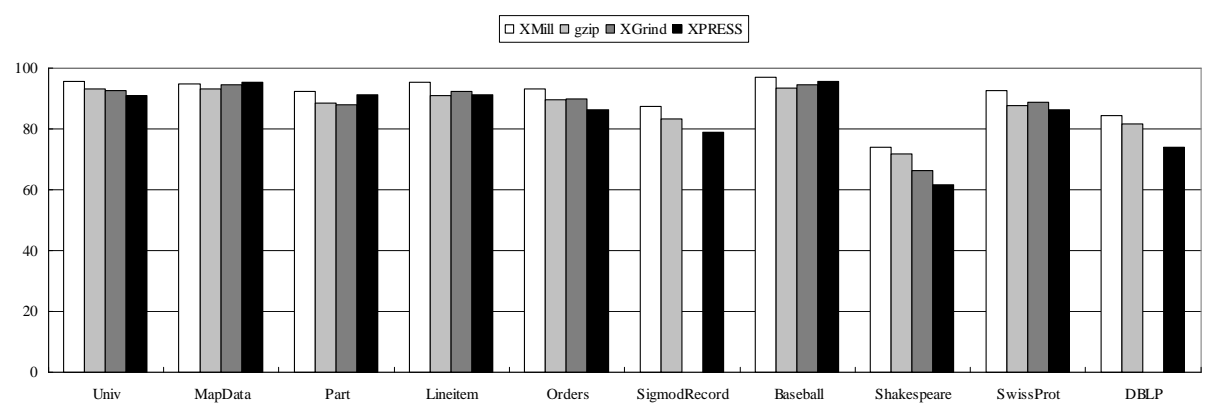

Fig. 18. Compression ratio after performing gzip

In addition, to show the effect of the built-in compression library zlib in XMill, we re-compressed the compressed files generated by XGrind and XPRESS using gzip which uses zlib internally. The result is shown in Figure 18. In Figure 18, as we expected, XMill still shows the best compression ratio. Since XMill groups semantically related data values into same containers, zlib effectively compresses XML data. However, the compression ratios of the re-compressed XML data by gzip are very close to that of XMill. Especially, for some data sets, the compression ratio of XPRESS for the re-compressed XML data is slightly higher than that of gzip. Thus, for archiving, applying gzip selectively for compressed XML data which is seldom queried is another alternative.

Although XMill shows the best performance in the compression ratio and the compression time, XMill does not support querying compressed XML data. Thus, to show the effectiveness of XPRESS, we compared the query performance of XPRESS to that of XGrind which supports querying compressed XML data and that of the XMill query engine which supports querying the uncompressed textual XML data.

We plotted the query processing cost of all queries for ten data sets in Figure 19. The query processing cost contains the result reconstruction time for all queries. Basically, Figure 19 (a) shows the query performance for small sized XML data sets (i.e., SigmodRecord, Part, Baseball, and Univ data sets), (b) is for medium sized XML data sets (i.e., Orders, MapData, and Shakespeare data sets), and (c) is for large sized XML data sets(i.e., Lineitem, SwissProt, and DBLP data sets), respectively. For each query, the three connected bars represent XMill, XGrind and XPRESS. Since XMill does not support direct querying on compressed XML data, XMill must completely decompress compressed XML data first before processing queries. Thus, the query processing cost for XMill is a sum of the complete decompression time and the actual query processing time. The complete decompression time consists of the decompression time of the compressed XML data and the creation time of the decompressed XML file. The actual query processing time 
consists of the parsing time of the decompressed XML file and the evaluation time of the given query. In addition, Table $\mathrm{V}$ shows the complete decompression time and query processing time of XMill for each data set in seconds. Under Query Processing Time, the values correspond to the time taken for the query types described in Table III. As shown in Figure 19, XPRESS outperforms XMill and XGrind over all cases.

\begin{tabular}{c|c|c|c|c|c|c|c}
\hline \multirow{2}{*}{ Data Set } & Decompression Time & \multicolumn{6}{|c}{ Query Processing Time(sec) } \\
\cline { 3 - 7 } & $(\mathrm{sec})$ & $\mathrm{T} 1$ & $\mathrm{~T} 2$ & $\mathrm{~T} 3$ & $\mathrm{~T} 4$ & $\mathrm{~T} 5$ & $\mathrm{~T} 6$ \\
\hline SigmodRecord & 0.02 & 0.31 & 0.19 & 0.09 & 0.16 & 0.35 & 0.24 \\
Part & 0.05 & 0.51 & 0.51 & 0.21 & 0.56 & 0.59 & 0.52 \\
Baseball & 0.07 & 0.55 & 0.39 & 0.37 & 0.43 & 0.38 & 0.43 \\
Univ & 0.17 & 1.70 & 1.58 & 0.79 & 1.50 & 1.92 & 1.54 \\
Orders & 0.43 & 4.06 & 4.16 & 1.69 & 3.46 & 3.75 & 4.02 \\
MapData & 0.28 & 0.73 & 0.94 & 0.55 & 0.89 & 1.02 & 1.12 \\
Shakespeare & 0.8 & 17.80 & 7.90 & 2.20 & 5.41 & 7.01 & 8.86 \\
Lineitem & 4.56 & 21.05 & 21.69 & 10.77 & 18.83 & 19.77 & 21.87 \\
SwissProt & 60.3 & 55.47 & 64.51 & 36.98 & 65.29 & 80.01 & 82.91 \\
DBLP & 75.12 & 82.81 & 85.73 & 39.57 & 63.01 & 71.56 & 79.97 \\
\hline
\end{tabular}

Table V. Query evaluation time of XMill

The query cost of query type 1 shows that the approximated reverse arithmetic encoder does not incur the degradation of efficiency. XPRESS is not very efficient for Shakespeare_1 because the query generates a large result. Since the lengths of path expressions in query type 2 are short, the query processing cost is low. Thus, the difference of query performance between XPRESS and XGrind is not conspicuous. However, the query performance of XPRESS for complicated path expressions (query type 3, 4, 5, and 6) is much better than those of XGrind and XMill since the query processor of XPRESS efficiently evaluates the queries using reverse arithmetic encoding. The advantage of using reverse arithmetic encoding appears for both simple queries and complex queries because the simple path is the building block of the complex query. Also, for the queries containing predicates, the performance gap increases since XPRESS minimizes the overhead of a partial decompression using order preserved encoders.

Of particular interest is the performance gap between query type 1,2 and query type $3,4,5,6$. Similar to the compression, the decompression time of XMill is the most efficient. Although XPRESS and XGrind evaluate the queries on the compressed data, the decompression of query results is required. As the size of query results decreases, the decompression overhead for query results of XPRESS and XGrind decreases. Therefore, the performance gap between XPRESS and XMill increases in the cases where queries contain predicates rather than simple/partial path expressions without predicates.

On the average, the query performance of XPRESS is 2.13 times better than that of XGrind and 4.31 times better than that of XMill, with the performance gap increased by the complexity of queries.

Lastly, we demonstrate the efficiency of the update processor of XPRESS. As mentioned previously, we compared the update performance of XPRESS to that of 


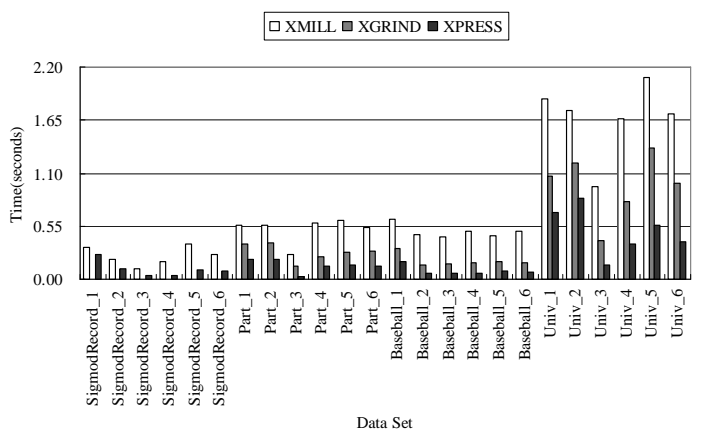

(a) SigmodRecord, Part, Baseball, and Univ Data Sets

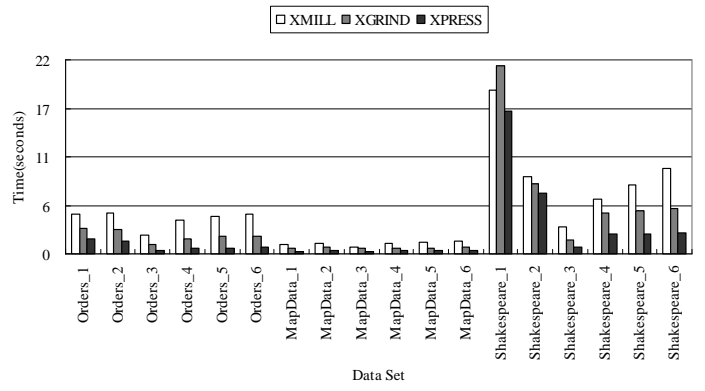

(b) Orders, MapData, and Shakespeare Data Sets

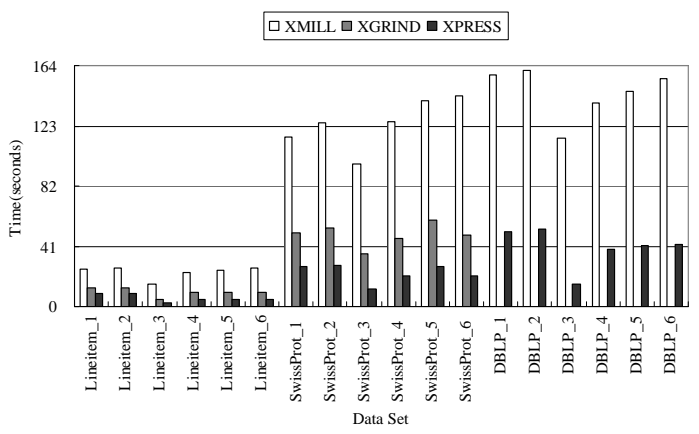

(c) Lineitem, SwissProt, and DBLP Data Sets

Fig. 19. Query evaluation time

a naive approach which decompresses compressed XML data using XMill, makes updates, and recompresses updated XML data using XMill. 


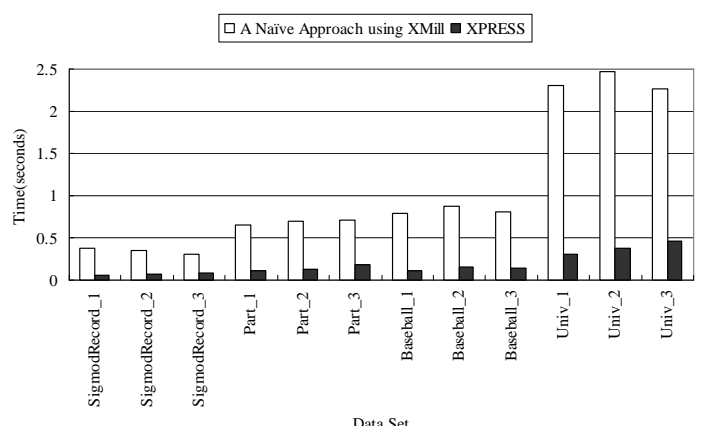

(a) SigmodRecord, Part, Baseball, and Univ Data Sets

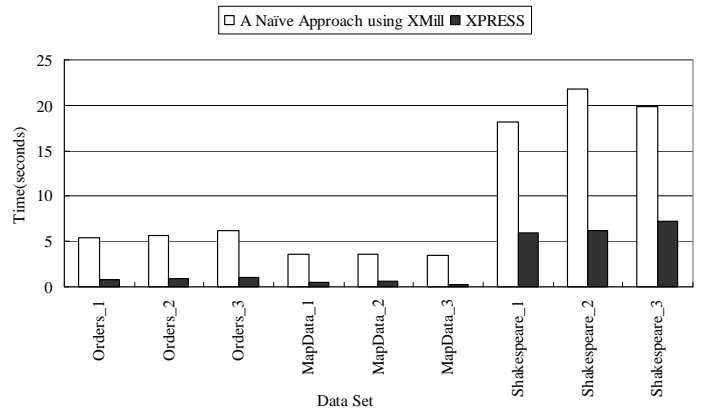

(b) Orders, MapData, and Shakespeare Data Sets

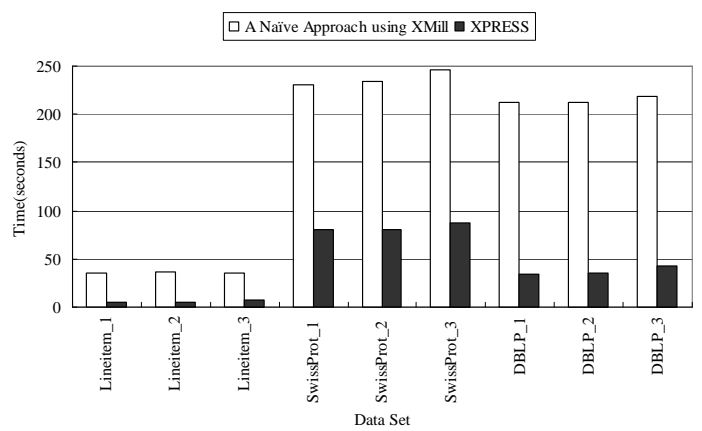

(c) Lineitem, SwissProt, and DBLP Data Sets

Fig. 20. Update time

We provided the processing cost of the updates whose representative examples ACM Transactions on Internet Technology, Vol. V, No. N, Month 20YY. 
are in Table IV.

In Figure 20, we divided the update cost with respect to sizes of XML data sets as presented for the query cost. The two connected bars in Figure 20 represent the naive approach with XMill and XPRESS, respectively.

As we expected, XPRESS outperforms the naive approach with XMill over all cases since the update processor of XPRESS does not perform the complete decompression of compressed XML data.

For update type 2, due to the insertion of the newly appeared tags, the recompression for the new encoded values for all the tags is required. Therefore, the updates of type 2 consume more time than those of type 1 . However, the update performance of XPRESS is considerably better than that of the naive approach with XMill since the updater of XPRESS performs only a partial decompression instead of the complete decompression, and the query performance of XPRESS is superior to that of the query engine for XMill. Similarly, the performance of XPRESS for the update type 3 is superior to that of the naive approach with XMill. As a result, the performance of the update processor of XPRESS is about 5.7 times faster than that of the naive approach with XMill.

In addition, we provided the updated portion of the updates presented in Figure 20 for the naive approach with XMill and XPRESS in Table VI. Type represents the types of updates as described in Table IV and Updated Size denotes the total size of the updated portion for the naive approach with XMill and XPRESS in bytes.

Consequently, XPRESS achieves significantly improved query performance compared to XGrind and shows a reasonable compression ratio. Also, XPRESS achieves significantly improved update performance compared to the naive approach with XMill.

\section{CONCLUSION}

In this paper, we propose XPRESS, an XML compressor which supports direct updates and efficient querying on compressed XML data. In XPRESS, we devise a novel encoding method, called reverse arithmetic encoding, which encodes a label path to a distinct interval in $[0.0,1.0)$. Using the containment relationships among the intervals, path expressions are evaluated on compressed XML data effectively. Furthermore, to save the disk space, we implement the approximated reverse arithmetic encoder which does not incur the loss of the accuracy and the efficiency. Also, to apply proper encoders for data values, we devise an efficient type inference engine and, by inferred type information, XPRESS encodes the data values. Since the encoders for numeric typed data values and the encoder for string typed data values do not lose the order information, we reduce the overhead of a partial decompression for range queries.

We implemented XPRESS, a compressor as well as a query processor and an update processor for compressed XML data. The query processor of XPRESS supports a wide class of XPath including order based and value based predicates. To show the efficiency of XPRESS, we conducted an extensive experimental study with real-life XML data sets. Experimental results show that XPRESS improves query performance. On the average, the query performance of XPRESS is 2.13 times 


\begin{tabular}{|c|c|c|c|}
\hline \multirow[t]{2}{*}{ Data Set } & \multirow[t]{2}{*}{ Type } & \multicolumn{2}{|c|}{ Updated Size(Bytes) } \\
\hline & & $\begin{array}{c}\text { naive approach } \\
\text { with XMill }\end{array}$ & XPRESS \\
\hline \multirow[t]{3}{*}{ SigmodRecord } & $\mathrm{T} 1$ & 402570 & 17892 \\
\hline & T2 & 415989 & 73059 \\
\hline & T3 & 69849 & 27702 \\
\hline \multirow[t]{3}{*}{ Part } & $\mathrm{T} 1$ & 273999 & 42000 \\
\hline & $\mathrm{T} 2$ & 558000 & 98000 \\
\hline & T3 & 54893 & 10000 \\
\hline \multirow[t]{3}{*}{ Baseball } & $\mathrm{T} 1$ & 167962 & 25746 \\
\hline & $\mathrm{T} 2$ & 342054 & 60074 \\
\hline & T3 & 22047 & 6130 \\
\hline \multirow[t]{3}{*}{ Univ } & $\mathrm{T} 1$ & 279075 & 41175 \\
\hline & $\mathrm{T} 2$ & 1276425 & 224175 \\
\hline & T3 & 110931 & 20582 \\
\hline \multirow[t]{3}{*}{ Orders } & $\mathrm{T} 1$ & 509999 & 52938 \\
\hline & $\mathrm{T} 2$ & 4185000 & 432327 \\
\hline & T3 & 462208 & 61761 \\
\hline \multirow[t]{3}{*}{ MapData } & $\mathrm{T} 1$ & 1800 & 70 \\
\hline & $\mathrm{T} 2$ & 1116 & 49 \\
\hline & T3 & 180 & 28 \\
\hline \multirow[t]{3}{*}{ Shakespeare } & $\mathrm{T} 1$ & 11757020 & 1923876 \\
\hline & $\mathrm{T} 2$ & 29820078 & 5237218 \\
\hline & T3 & 5685014 & 2956429 \\
\hline \multirow[t]{3}{*}{ Lineitem } & $\mathrm{T} 1$ & 7160824 & 1263675 \\
\hline & $\mathrm{T} 2$ & 16788825 & 2948575 \\
\hline & T3 & 1854326 & 421225 \\
\hline \multirow[t]{3}{*}{ SwissProt } & $\mathrm{T} 1$ & 62526526 & 9584358 \\
\hline & $\mathrm{T} 2$ & 127335042 & 22363502 \\
\hline & T3 & 10204644 & 5669208 \\
\hline \multirow[t]{3}{*}{ DBLP } & $\mathrm{T} 1$ & 41392845 & 7004943 \\
\hline & $\mathrm{T} 2$ & 59224167 & 10401279 \\
\hline & T3 & 16873191 & 9710766 \\
\hline
\end{tabular}

Table VI. Updating portion of the updates

better than that of XGrind and 4.31 times better than that of XMill, with the performance gap increasing with the complexity of queries. The average compression ratio of XPRESS is $71 \%$. Also, we demonstrated the efficiency of the update performance of XPRESS by comparing with that of a naive approach using XMill.

Currently, the type inference engine of XPRESS distinguishes the numeric data and textual data. Thus, for our future work, we plan to extent XPRESS to support complex typed data values such as URI (Uniform Resouce Identifier) using data mining algorithms.

\section{REFERENCES}

Aboulnaga, A., Alameldeen, A. R., and Naughton, J. F. 2001. Estimating the selectivity of xml path expressions for internet scale applications. In Proceedings of 27th International Conference on Very Large Data Bases. 591-600.

Arion, A., Bonifati, A., Costa, G., D'Aguanno, S., Manolescu, I., and Pugliese, A. 2004. Efficient query evaluation over compressed xml data. In Proceedings of 9th International Conference on Extending Database Technology. 200-218.

Bayardo, R. J., Gruhl, D., Josifovski, V., and Myllymaki, J. 2004. An evaluation of binary ACM Transactions on Internet Technology, Vol. V, No. N, Month 20YY. 
xml encoding optimizations for fast stream based xml processing. In Proceedings of WWW2004. $17-22$.

Boag, S., Chamberlin, D., Fernandez, M. F., Florescu, D., Robie, J., and Simeon, J. 2002. XQuery 1.0: An XML Query Language. Working Draft, http://www.w3.org/TR/2002/WDxquery-20020816.

Bray, T., Paoli, J., Sperberg-McQueen, C. M., and Maler, E. 1998. Extensible Markup Language (XML) 1.0. W3C Recommendation, http://www.w3.org/TR/REC-xml.

Chen, Z., Gehrke, J., And Korn, F. 2000. Query optimization in compressed database systems. In Proceedings of ACM SIGMOD.

Cheng, J. And NG, W. 2004. Xqzip: Querying compressed xml using structural indexing. In Proceedings of 9th International Conference on Extending Database Technology. 219-236.

Clark, J. and DeRose, S. 1999. XML Path Language(XPath) Version 1.0. W3C Recommendation, http://www.w3.org/TR/xpath.

Cover, R. 2001. The XML Cover Pages. http://www.oasis-open.org/cover/xml.html.

ETRI. GML Data. http://www.telematics.re.kr/board/fboard/proc/fboard_list.jsp? code $=$ tech $\& \mathrm{md} 1=4 \& \mathrm{md} 2=3 \& \mathrm{md} 3=1$.

FERnANDEZ, M. F. AND SUCIU, D. 1998. Optimizing regular path expressions using graph schemas. In Proceedings of the 14th International Conference on Data Engineering. 14-23.

Fernandez, M. F., TAN, W. C., And Suciu, D. 2000. Silkroute: trading between relations and xml. WWW9/Computer Networks 33, 1-6 (June), 723-745.

Florescu, D. and Kossman, D. 1999. Storing and querying xml data using an rdmbs. IEEE Data Engineering Bulletin 22, 3 (Sept.), 27-34.

Goldman, R. And Widom, J. 1997. Dataguides: Enable query formulation and optimization in semistructured databases. In Proceedings of 23rd International Conference on Very Large Data Bases. 436-445.

Grust, T. 2002. Accelerating xpath location steps. In Proceedings of the 2002 ACM SIGMOD International Conference on Management of Data. 109-120.

Harold, E. R. Long Baseball Examples from The XML Bible. ibiblio, http://www.ibiblio.org/xml/books/biblegold/examples/baseball/.

Howard, P. G. And Vitter, J. S. 1991. Analysis of arithmetic coding for data compression. In Proceedings of the IEEE Data Compression Conference. 3-12.

Huffman, D. A. 1952. A method for the construction of minimum redandancy codes. In Proceedings of the Institute of Radio Engineers 40. 1098-1101.

LI, Q. AND Moon, B. 2001. Indexing and querying xml data for regular path expressions. In Proceedings of 27th International Conference on Very Large Data Bases. 361-370.

Liefke, H. And Suciu, D. 2000. Xmill: An efficient compressor for xml data. In Proceedings of the 2000 ACM SIGMOD International Conference on Management of Data. 153-164.

Open GIS CONSORTIUm. http://opengis.net/gml/.

Salminen, A. And Tompa, F. W. 1992. Pat expressions: an algebra for text search. Acta Linguistica Hungarica 41, 1-4, 277-306.

Salomon, D. 1998. Data Compression, the complete reference. Springer-Verlag New York, Inc.

Shanmugasundaram, J., Shekita, E. J., Barr, R., Carey, M. J., Lindsay, B. G., Pirahesh, H., AND Reinwald, B. 2000. Efficiently publishing relational data as xml documents. In Proceedings of 26th International Conference on Very Large Data Bases. 65-76.

Shannon, C. E. 1948. A mathematical theory of communication. Bell Syst. Tech. J. 27, 398-403.

Shimura, T., Yoshikawa, M., and Uemura, S. 1999. Storing and retrieval of xml documents using object-relational databases. In Proceedings of 10th International Conference, DEXA. 206-217.

Tatarinov, I., Ives, Z. G., Halevy, A. Y., And Weld, D. S. 2001. Updating xml. In Proceedings of the 2001 ACM SIGMOD International Conference on Management of Data.

Tatarinov, I., Viglas, S. D., Beyer, K., Shanmugasundaram, J., Shekita, E., and Zhang, C. 2002. Storing and querying ordered $\mathrm{xml}$ using a relational database system. In Proceedings of the 2002 ACM SIGMOD International Conference on Management of Data. 204-215. 
Tolani, P. M. And Haritsa, J. R. 2002. Xgrind: A query-friendly xml compressor. In Proceedings of 18th International Conference on Data Engineering. 225-234.

UW. XML Data Repository. http://www.cs.washington.edu/research/xmldatasets/www/repository.html. Witten, I. H., Neal, R. M., And Cleary, J. G. 1987. Arithmetic coding for data compression. Communications of the ACM 30, 6 (June), 520-540.

Zhang, N., KaCholia, V., And Özsu, M. T. 2004. A succinct physical storage scheme for efficient evaluation of path queries in xml. In Proceedings of the 20th International Conference on Data Engineering. 54-65. 